RESEARCH

\title{
Tumor induced osteomalacia in head and neck region: single center experience and systematic review
}

\author{
Ravikumar Shah ${ }^{1}$, Anurag R Lila ${ }^{1}$, Swati Ramteke-Jadhav'1, Virendra Patil', Abhishek Mahajan'2, Sushil Sonawane', \\ Puja Thadani ${ }^{1}$, Anil Dcruz ${ }^{3}$, Prathamesh Pai ${ }^{3}$, Munita Bal ${ }^{4}$, Subhada Kane ${ }^{4}$, Nalini Shah ${ }^{1}$ and Tushar Bandgar ${ }^{1}$ \\ ${ }^{1}$ Department of Endocrinology, Seth GS Medical College \& KEM Hospital, Parel, Mumbai, India \\ ${ }^{2}$ Department of Radiodiagnosis and Imaging, Tata Memorial Hospital, Mumbai, Maharashtra, India \\ ${ }^{3}$ Department of Head Neck Surgery, Tata Memorial Hospital, Mumbai, Maharashtra, India \\ ${ }^{4}$ Department of Pathology, Tata Memorial Hospital, Mumbai, Maharashtra, India \\ Correspondence should be addressed to A R Lila: anuraglila@gmail.com
}

\begin{abstract}
Tumor-induced osteomalacia in the head and neck region remains a challenging diagnosis to manage. Literature pertaining to management and outcome details remains sparse. We describe two cohorts: cohort 1 included seven patients from a single center in Western India with tumors located in paranasal sinuses $(n=3)$, intracranial $(n=2)$ and maxilla $(n=2)$. The unique features from our series is the management of persistent disease with radiation therapy $(n=2)$ and peptide receptor radionuclide therapy (PRRT) $(n=1)$. Cohort two has 163 patients identified from 109 publications for systematic review. Paranasal sinuses, mandible, intracranial disease, maxilla and oral cavity, in descending order, are reportedly common tumor sites. Within this cohort, mean age was $46 \pm 14$ years at presentation with $44.1 \%$ having local symptoms. Duration of symptoms varied from 1 to 240 months. Pre-surgery mean serum phosphorus was $1.4 \pm 0.4 \mathrm{mg} / \mathrm{dL}$ and median FGF-23 levels were 3.6 (IQR:1.8-6.8) times of normal upper limit of normal. Majority $(97.5 \%)$ were managed primarily with surgical excision; however, primary radiotherapy $(n=2)$ and surgery combined with radiotherapy $(n=2)$ were also reported. Twenty patients had persistent disease while nine patients had recurrence, more commonly noted with intracranial and oral cavity tumors. Surgery was the most common second mode of treatment employed succeeded by radiotherapy. Four patients had metastatic disease. The most common histopathological diagnosis reported is PMT mixed connective tissue, while the newer terminology 'PMT mixed epithelial and connective tissue type' has been described in 15 patients.
\end{abstract}

\section{Key Words}

- tumor-induced osteomalacia (TIO)

- oncogenic osteomalacia

- head and neck

- systematic review

\section{Introduction}

Tumor-induced osteomalacia (TIO), also known as oncogenic osteomalacia, is a rare paraneoplastic syndrome caused by overproduction of fibroblast growth factor 23 (FGF23) by a tumor. FGF-23 plays a vital role in renal phosphate handling and vitamin D synthesis. Hence, TIO is characterized by hypophosphatemia due to renal phosphate wasting, inappropriately normal or low 1,25 dihydroxy vitamin $\mathrm{D}$, and elevated or inappropriately normal plasma FGF-23. These biochemical alterations eventually result in osteomalacia. Due to its rarity, the diagnosis of TIO is delayed with the average time from onset of symptoms to diagnosis being more than 2.5 years (1). As a result, patients often present in a debilitated state with multiple fractures, severe muscle weakness https://ec.bioscientifica.com https://doi.org/10.1530/EC-19-0341

(c) 2019 The authors Published by Bioscientifica Ltd

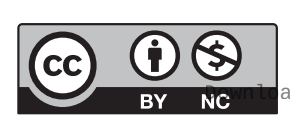

This work is licensed under a Creative Commons Attribution-NonCommercial 4.0 International License. ded from Bioscientifica.com at 04/26/2023 02:16:47PM 
and loss of height due to skeletal deformities. Even with a high index of suspicion, tumor localization remains challenging as the offending tumor may be very small and can be anywhere in the body. Complete tumor resection remains the mainstay of treatment and is known to result in dramatic resolution of symptoms.

The first case of TIO was reported by Robert McCance in 1947 who treated a patient having low phosphorus levels and bone pain with high doses of vitamin $\mathrm{D}$ suspecting her to be a case of 'vitamin D resistance'; however, the symptoms did not completely resolve until a tumor found in the femur was removed (2). Thereafter, more than 300 cases of TIO have been reported in literature with more than 200 being reported since 2000 (3). The most common tumor site is the lower extremity (>40\%) followed by the head and neck region (>20\%) (4). There have been several reviews on pathological characters of such tumors but there is no comprehensive review describing clinical characteristics and management of patients with TIO in head and neck region. This article aims to describe a single-center experience with TIO involving the head and neck region followed by a comprehensive clinically oriented review of world literature for the same.

\section{Materials and methods}

\section{Cohort 1}

Medical records of patients attending Department of Endocrinology, KEM Hospital, Mumbai who were diagnosed with TIO from January 2005 till August 2018 were reviewed after obtaining approval from Institutional Ethical Committee II, Seth G S Medical college and KEM Hospital, Mumbai. Informed consent for the photographs, publication of their clinical details and/or imaging was taken. Patients diagnosed with TIO involving the head and neck region were identified and reviewed for inclusion. Concurrently, patients diagnosed with TIO in other regions, and patients with secondary TIO (3) (including neurofibromatosis, epidermal nevus syndrome, and polyostotic fibrous dysplasia of bone) were excluded from the study.

Diagnosis of TIO was considered in patients presenting with features of hypophosphatemia in absence of relevant family history, evidence of renal phosphate wasting (as demonstrated by low \% fractional tubular reabsorption of phosphate (TRP) and tubular maximum for phosphate corrected for glomerular filtration rate (TMP/GFR)) with elevated fibroblast growth factor-23 (FGF-23). Only those patients who had anatomical/functional imaging (CT/MRI or Ga-DOTATATE PET/CT) demonstrating localization of tumor in head and neck region have been included for analysis $(n=7)$.

Biochemical parameters recorded pre-operatively include S. calcium, S. phosphorus, S. alkaline phosphatase (ALP), TMP/GFR, TRP and FGF23 levels, and postoperatively include S. phosphorus and FGF-23 levels. Normal ranges for various parameters at our institute are as follows: S. calcium (9-10.5 mg/dL), S. phosphorus (2.5-5 mg/dL), S. ALP (<117 U/L), TMP/GFR (age- and sex-adjusted values as recommended by Chong et al. (3)), TRP (>85\%) and C-terminal FGF-23 (0-150RU/mL). Furthermore, details from imaging studies done for localization (CT or Ga-DOTATATE PET/CT), treatment modality used, and histopathology reports have been included for analysis. For patients having recurrent disease additional information including time of recurrence following primary management, biochemical profile, localization of recurrent disease and secondary modality of treatment used was documented.

Tubular resorption of phosphate was measured from phosphate and creatinine levels in a spot fasting urine and serum samples at baseline before starting phosphate supplements. TMP/GFR was calculated with use of a nomogram reported by Bijvoet et al. FGF23 was assessed by enzyme-linked immunosorbent assay (FGF23 (C-terminal) kit, Immunotopics, Inc, San Clemente, CA, USA). The kit has sensitivity, an intra-assay coefficient of variation $(\mathrm{CV})$, and an inter-assay $\mathrm{CV}$ of $30 \mathrm{RU} / \mathrm{mL}$, 5 and $7.3 \%$, respectively. Serum 1,25(OH)2 vitamin D was assessed by radioimmunoassay (RIA), using a DIA source RIA CT kit by DIA source Immunoassays, SA, with an intra-assay CV of $4.5-9.3 \%$ (at 77.3 and $24.5 \mathrm{ng} / \mathrm{L}$ concentrations, respectively) and inter-assay CV of $11.3-12.7 \%$ (at 33.4 and $13.6 \mathrm{ng} / \mathrm{L}$ concentrations, respectively). Whole-body (head to toe) scanning with two acquisitions were obtained $1-1.5 \mathrm{~h}$ post intravenous injection of 74-111 MBq of DOTATATE labeled with 68Ga. $68 \mathrm{Ga}$ was obtained from an in-house $68 \mathrm{Ge} / 68 \mathrm{Ga}$ generator. Scans were acquired on a GE Discovery STE PET/CT with $128 \times 128$ matrix size and 3 min per bed position of iterative algorithm time. The numbers of bed positions were dependent on the height of the patient, usually 10-12 per patient. CT scans were obtained on a 64-slice Phillips Brilliance CT scanner, while MRI scans were performed on a 1.5 tesla Siemens Sonata (Henkestrabe, Germany) MR scanner.
This work is licensed under a Creative Commons Attribution-NonCommercial 4.0 International License. ded from Bioscientifica.com at 04/26/2023 02:16:47PM via free access 


\section{Cohort 2}

We searched for all original and review articles in PubMed till June 2019 (Fig. 1). Individual search was carried out for terms 'Tumour-Induced Osteomalacia', 'Oncogenic Osteomalacia', and 'Phosphaturic Mesenchymal Tumour'. All original and review articles published in English were reviewed for inclusion. Only publications describing TIO in head and neck region were included. A secondary search for relevant publications was carried out by handsearching through the reference lists of selected publications. Hence, in addition to the cases described in our series, we reviewed 163 index cases from 109 publications of TIO of head and neck region previously reported in literature. Clinical profile, biochemical investigations, imaging modality used for localization, location of tumor, treatment modalities used, histopathology findings, recurrence and its management, and metastasis if any were noted. Whenever serum levels of calcium, phosphorus, parathyroid hormone (PTH), 1,25 (OH)2 vitamin D3 levels were available in SI units, they were converted to conventional units with online calculators for uniformity in documentation. Serum ALP when available in units/liter only was included for analysis, while values reported in any other units were excluded due to non-availability of a suitable conversion method.

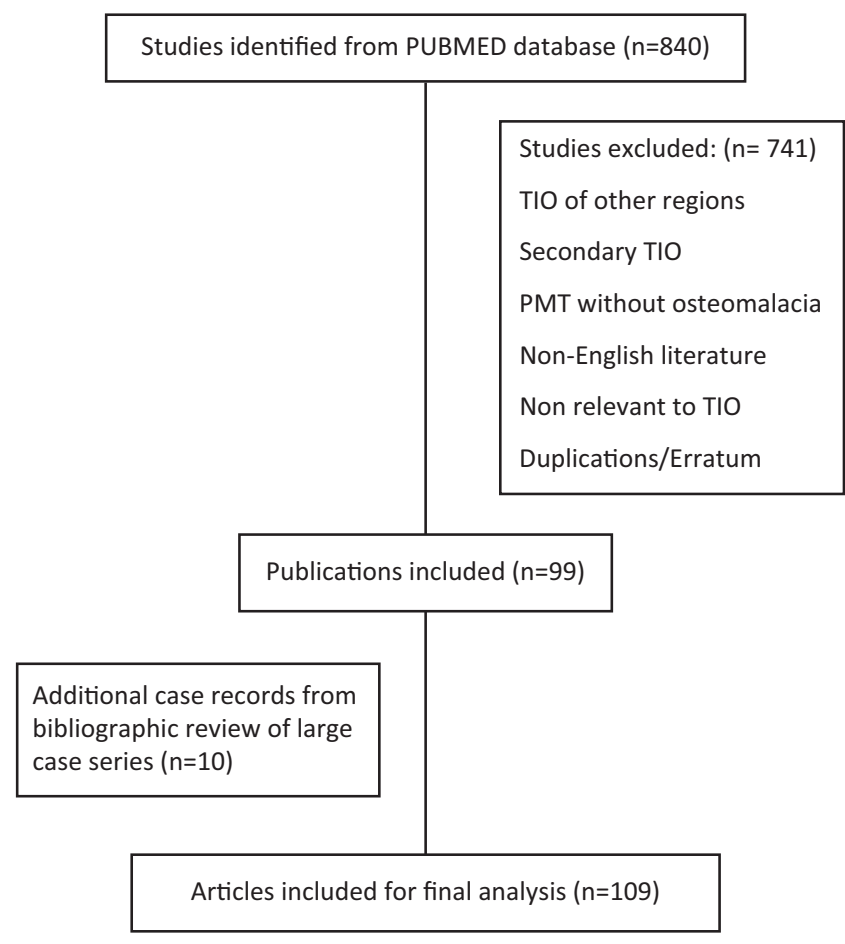

Figure 1

Flowchart of search strategy and selection of studies for inclusion in systematic review.

\section{Statistical analysis}

Statistical analysis was performed using SPSS software version 23.0. Mean ( \pm standard deviation (s.D.)) was used for continuous variables when they were normally distributed and median (interquartile range (IQR)) was used for variables with skewed distribution. The difference between categorical variables was analyzed using chisquare test. $P$ value $<0.05$ is considered significant.

\section{Results}

\section{Cohort 1}

This cohort includes seven index patients with TIO involving head and neck region. Their characteristics are described in Table 1 . The cohort comprised four males and three females with mean age of $42.7 \pm 10.6$ years whose tumors were located in paranasal sinuses $(n=3)$, maxilla $(n=2)$, and intra-cranially $(n=2)$. All patients presented with bone pain and muscle weakness, while pathologic fractures $(n=4)$ and local symptoms $(n=5)$ were present in majority of patients. The time lag from onset of symptoms to diagnosis was lengthy (mean: $65.1 \pm 50.3$ months). In four patients, location of tumor was suspected at initial presentation based on clinical history and examination. Thereafter, tumor location was confirmed with Ga-DOTANOC in two patients, with MRI in one patient and CT in one patient. Three patients were primarily detected on Ga-DOTANOC/DOTATATE PET/CT; one patient had a history of epistaxis elicited retrospectively after tumor localization. Mean tumor size was $3.6 \pm 1.3 \mathrm{~cm}$. Except for one patient (who was initially operated at another hospital), pre and post-operative serum phosphorus and FGF-23 levels were available in all patients (Table 1). Three patients were cured with initial surgery, while four had persistent disease. No recurrence was documented in patients cured initially $(n=3)$ over a mean follow-up of 17 months. Out of four patients with persistent disease, one patient was cured with repeat surgery only, two patients were cured with repeat surgery and external beam radiation therapy (EBRT), and one has stable disease after peptide receptor radionuclide therapy (PRRT). Histopathologic findings revealed phosphaturic mesenchymal tumor mixed connective tissue type (PMTMCT) in four patients, while the remaining three patients had PMT-OF (ossifying fibroma like), hemangiopericytoma, and odontogenic fibroma, respectively. Clinical images of case numbers one, five and six are shown in Figs 2, 3 and 4 respectively.

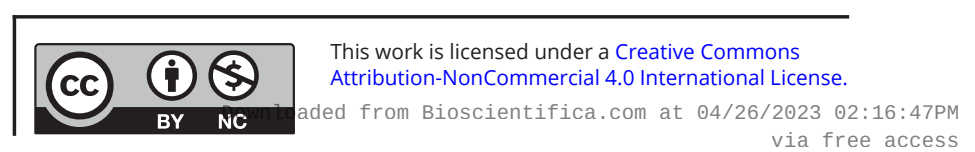




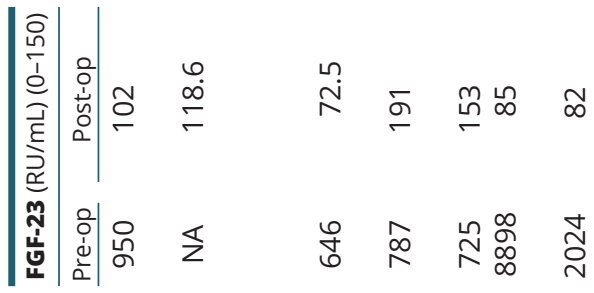

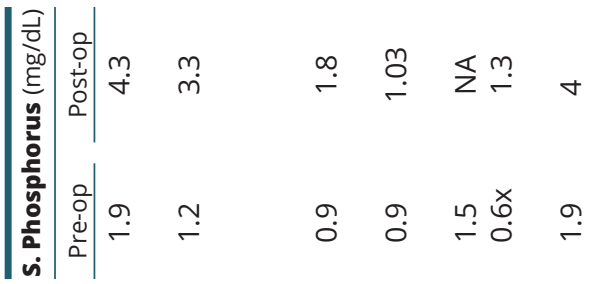

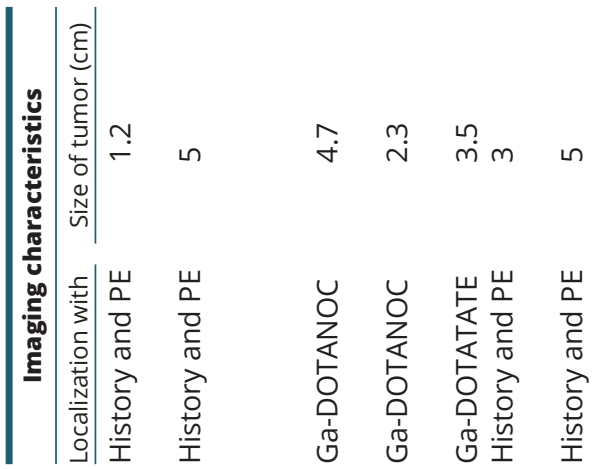

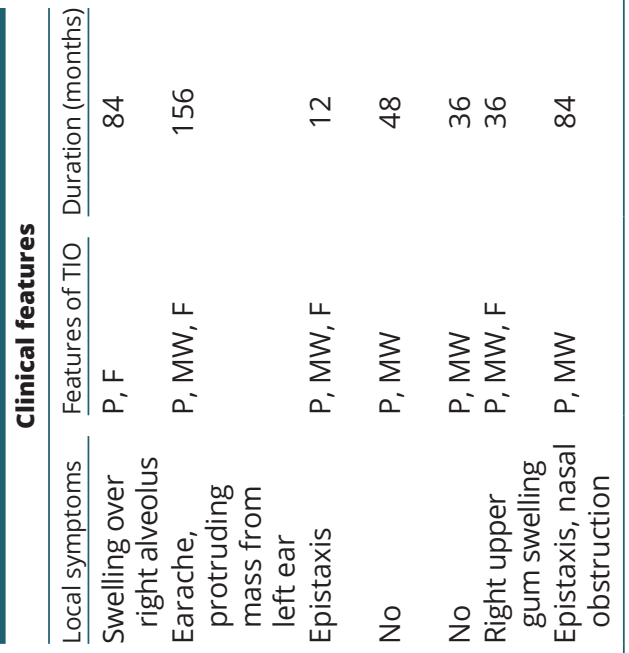

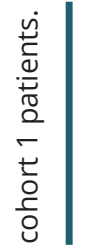

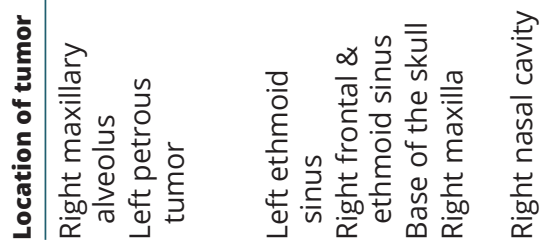

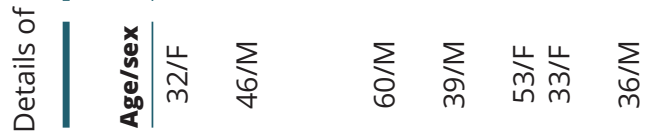

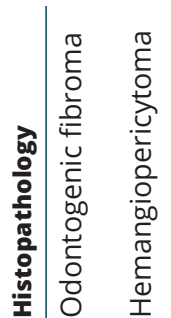

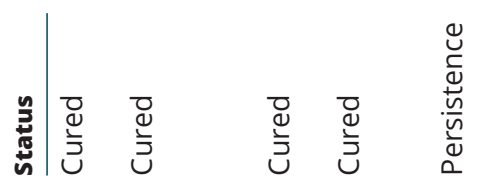
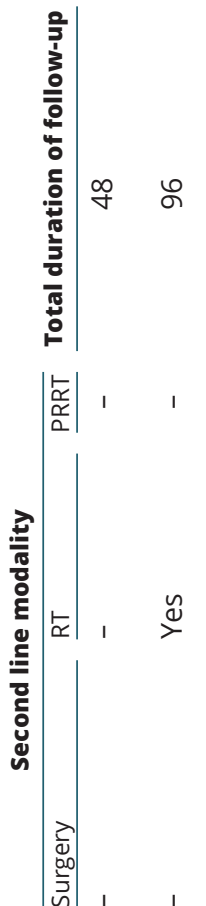

$\stackrel{m}{m}$

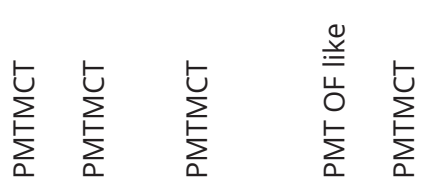

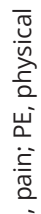
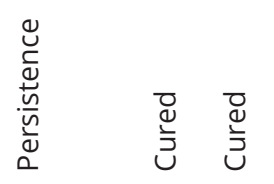

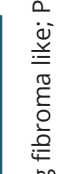

离

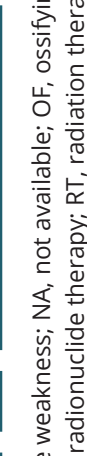

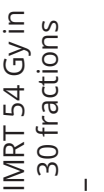
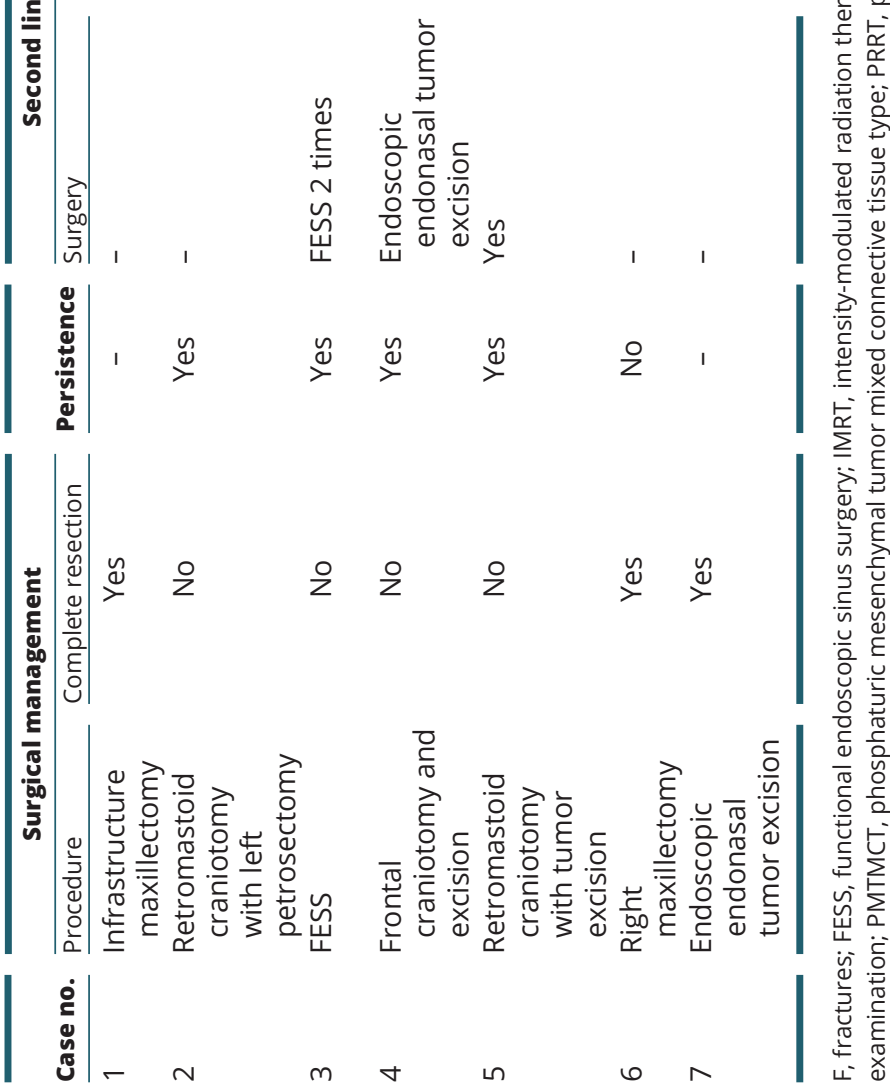

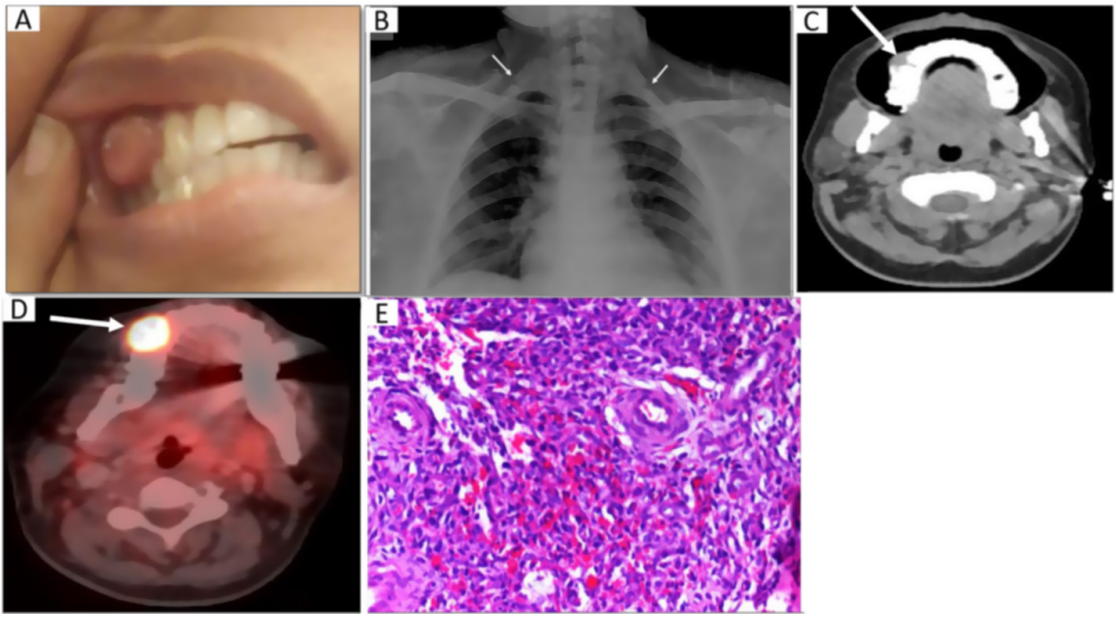

Figure 2

(Case 1): A 32-year-old female presented with bone pains and multiple fractures for 7 years. On examination, approximately $2 \mathrm{~cm}$-sized round swelling in right upper alveolus was seen (A). Preoperative chest radiograph (image contrast adjusted) showing Looser's zone along lateral border of scapula (arrows) suggestive of osteomalacia (B). Axial contrast-enhanced CT image soft tissue window showing small enhancing lesion in right upper alveolus (arrow) extending from canine to 1 st molar tooth causing erosion of right upper alveolus (C). Ga-DOTATATE PET scan showing increased uptake at the level of right maxillary alveolus (arrow) (D). After excision histopathological examination showing tumor comprising of spindle cells with scattered osteoclastic giant cells bearing histologic semblance to giant cell granuloma (odontogenic fibroma) (E) (H\&E, 400×).

\section{Cohort 2}

This cohort consists of 163 index patients from 109 publications. Pertinent data relevant to index patients is provided in Table 2. Details of clinically relevant parameters are summarized in Table 3. Tests done using two different methods have been tabulated separately in Table 3. Due to heterogeneity in reporting of various parameters, the number of cases included (as denominator) have been specified for each parameter. The mean age was $46 \pm 14$ years with equal male:female ratio. The reported frequency of tumor sites, in descending order, are paranasal sinuses, mandible, intracranial,

maxilla, oral cavity and others. Approximately half the patients (44.1\%) had evident local symptoms. Bone pain and muscle weakness were most commonly reported. Late complications of hypophosphatemia such as fractures (61\%) and bony deformities including kyphosis/scoliosis with resultant height loss (25.7\%) were seen in a significant number of patients. Most patients were diagnosed late in their disease course, despite early access to health care, with median duration from symptom onset being almost 4 years. Out of 163 patients, median elevation of FGF-23 up to 3.6 times ULN has been reported in 55 patients with the interquartile range (IQR) being $1.8-6.8 \times$ ULN . The primary treatment modality was surgery in most

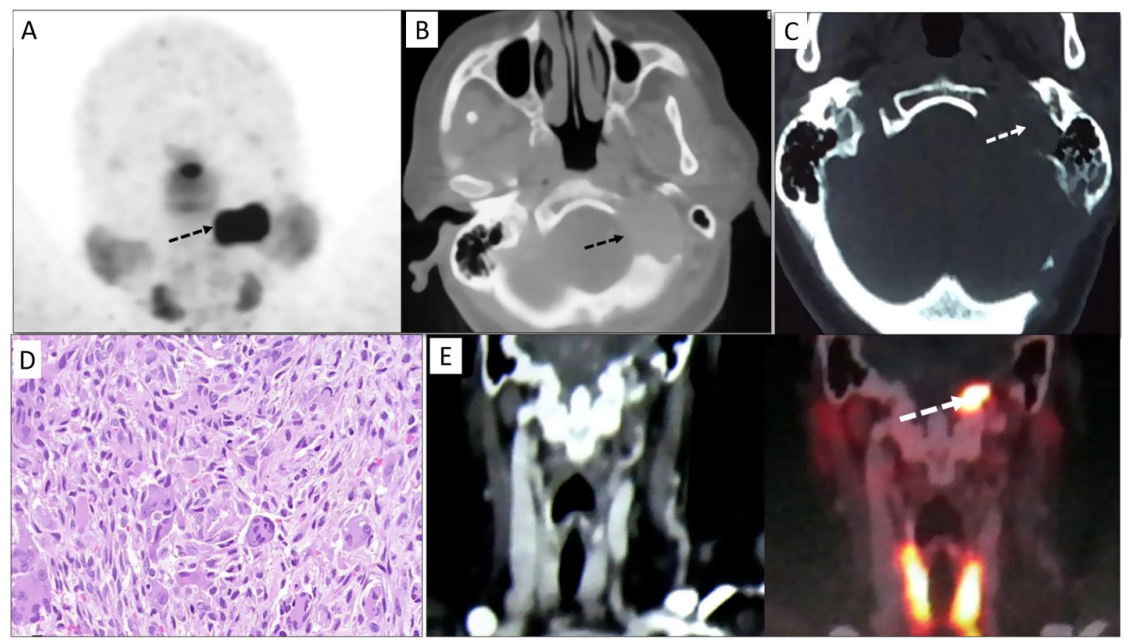

Figure 3

(Case 5): A 53-year-old female presenting with pain in bilateral groins and difficulty in walking for 3-year duration. As investigations confirmed the diagnosis of FGF-23-dependent

hypophosphatemic osteomalacia, ${ }^{68} \mathrm{Ga}$-DOTATATE PET scan was done to locate the tumor which showed increased uptake in base of skull in left side (dashed arrows) (A). Corresponding axial CT images (B) showing soft tissue density lesion involving occipital bone on left side with erosion of the mastoid and petrous part of adjacent temporal bone. Retromastoid craniotomy with tumor excision was done. Histopathological examination showed hypercellular tumor composed of prominent small blood vessels with areas of hemorrhage (H\&E, 200x) (D). Post first surgery repeat ${ }^{68} \mathrm{Ga}$-DOTATATE scan and corresponding $\mathrm{CT}$ images showing residual uptake in base of skull in left side (dashed arrows) in the soft tissue density lesion involving occipital bone on left side with erosion of the mastoid and petrous part of adjacent temporal bone (E). After failed second surgery, patient is now having stable disease after two cycles of PRRT.

https://ec.bioscientifica.com https://doi.org/10.1530/EC-19-0341 (c) 2019 The authors Published by Bioscientifica Ltd

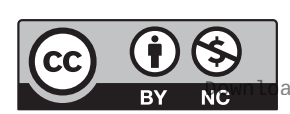

This work is licensed under a Creative Commons Attribution-NonCommercial 4.0 International License. ded from Bioscientifica.com at 04/26/2023 02:16:47PM 


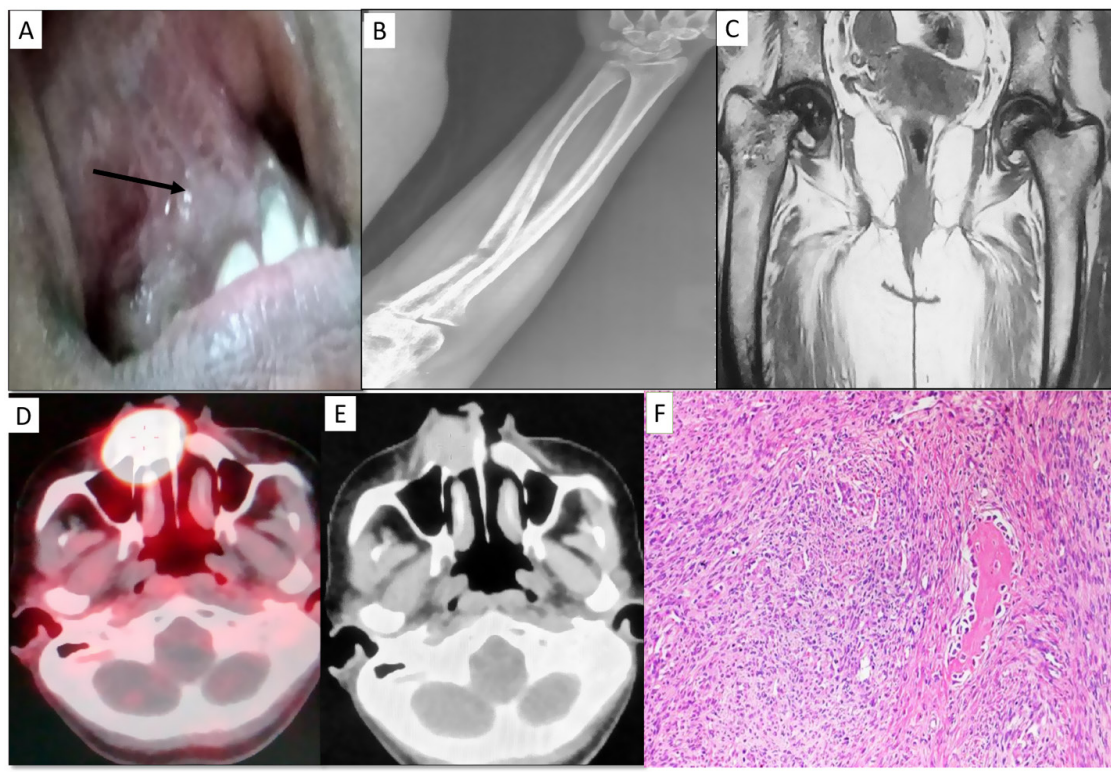

\section{Figure 4}

(Case 6): A 33-year-old female presenting with pain in bilateral groins, difficulty in walking and multiple fractures for 3-year duration. There was past history of dental surgery for some 'gum swelling'. On examination, there was swelling in right upper alveolar region (A). X-ray right forearm AP view (image contrast adjusted) showing ulnar shaft fracture (B). MRI hip showing bilateral femoral neck insufficiency fractures which was reported as 'bilateral avascular necrosis' (C). Ga-DOTANOC scan showing uptake in the right maxillary tumor (D). CECT PNS axial view showing $3 \mathrm{~cm}$ tumor in right maxillary region (E). Patient was cured with right maxillectomy and osseous reconstruction. Histopathology showed tumor composed of cellular connective tissue intermixed with woven bone displaying osteoblastic rimming (i.e. ossifying fibroma-like histology) (H\&E, 100×).

patients (97.5\%). Two patients with intracranial tumors, who declined surgery, were treated with primary EBRT. Also, two patients received immediate post-operative EBRT for prevention of recurrence due to fear of incomplete tumor removal.

Out of 148 patients for whom outcome data were available; 119 patients had complete initial response to surgery, 20 patients had persistent disease and 9 patients had recurrence as defined by worsening of post-operatively documented normal biochemistry over a variable period of 2-204 months. Patients with persistent/recurrent disease $(n=29)$ were predominantly managed with surgery $(65.3 \%)$ and/or radiotherapy (30.7\%). Among these patients 11 were reported to be alive with no evidence of disease (ANED) and remaining patients were managed with phosphorus supplements with/without other treatment modalities. Four patients had metastatic disease with lymph node and/or lung metastasis. Histopathologically, PMTMCT (48.7\%) remains the most commonly reported tumor type followed by hemangiopericytoma (22.7\%), PMT of mixed epithelial and connective tissue type (9.4\%), giant cell tumor (3.1\%) and odontogenic fibroma (3.1\%). Other rare types of tumor have been shown in Table 3.

\section{Discussion}

TIO is a rare and underreported condition due to unawareness about the characteristic clinical and biochemical profile among treating clinicians. Through this study, we aim to highlight our experience with TIO cases involving head and neck region and provide a review of published literature analyzed on a per-patient basis. This will increase awareness and provide valuable insight on critical management issues for this rare diagnosis.

\section{Cohort 1}

A significant time gap between initial presentation till diagnosis persists even in the presence of local symptoms (1). For any atypical head and neck mass, clinician should enquire into history relevant to osteomalacia, and for a symptomatic patient appropriate biochemistry (S. calcium, S. phosphorus and alkaline phosphatase) should be requested. Vice versa, in a patient with nonlocalized TIO, a clinician should examine oral and nasal cavities for palpable swellings and enquire about relevant local symptoms.

At our center we carry out a complete biochemical evaluation for TIO which includes calcium studies (S. calcium, S. phosphorus, ALP), TMP/GFR, 1,25 (OH) vitamin D3 and FGF-23 levels. FGF-23 serves as a diagnostic marker as well as an indicator of residual disease or recurrence during long-term follow-up. Thereafter, functional imaging with Ga-DOTANOC PET/CT for localization is done. Its superiority compared to FDG-PET/CT is well established $(110,111,112)$. Functional imaging is followed by appropriate anatomic imaging to determine tumor extent and plan for surgical management. Alternatively, in a TIO patient presenting with local symptoms or a mass in head and neck region, anatomic imaging (CT/MRI) followed by biopsy can also be used. 

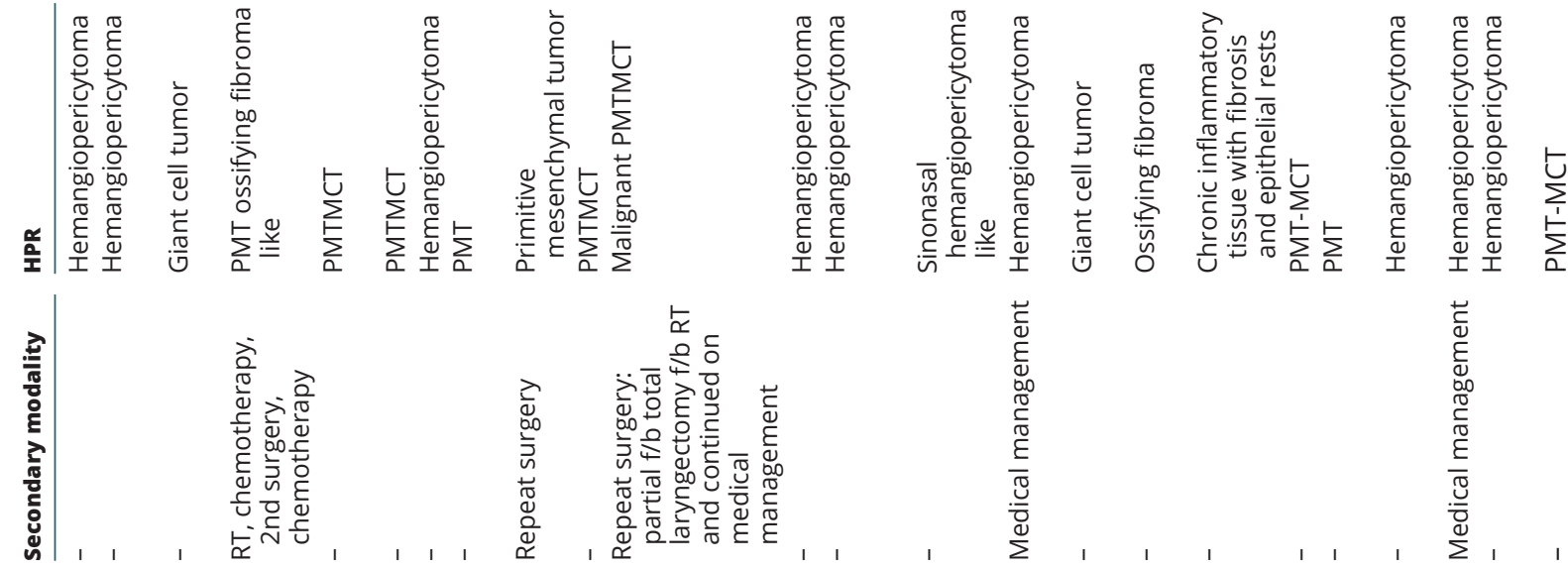

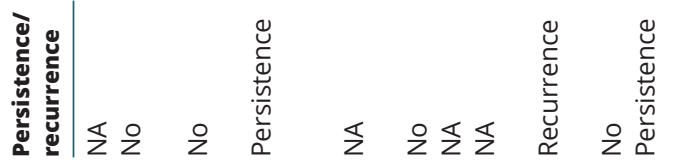

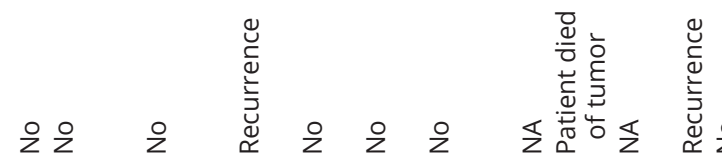
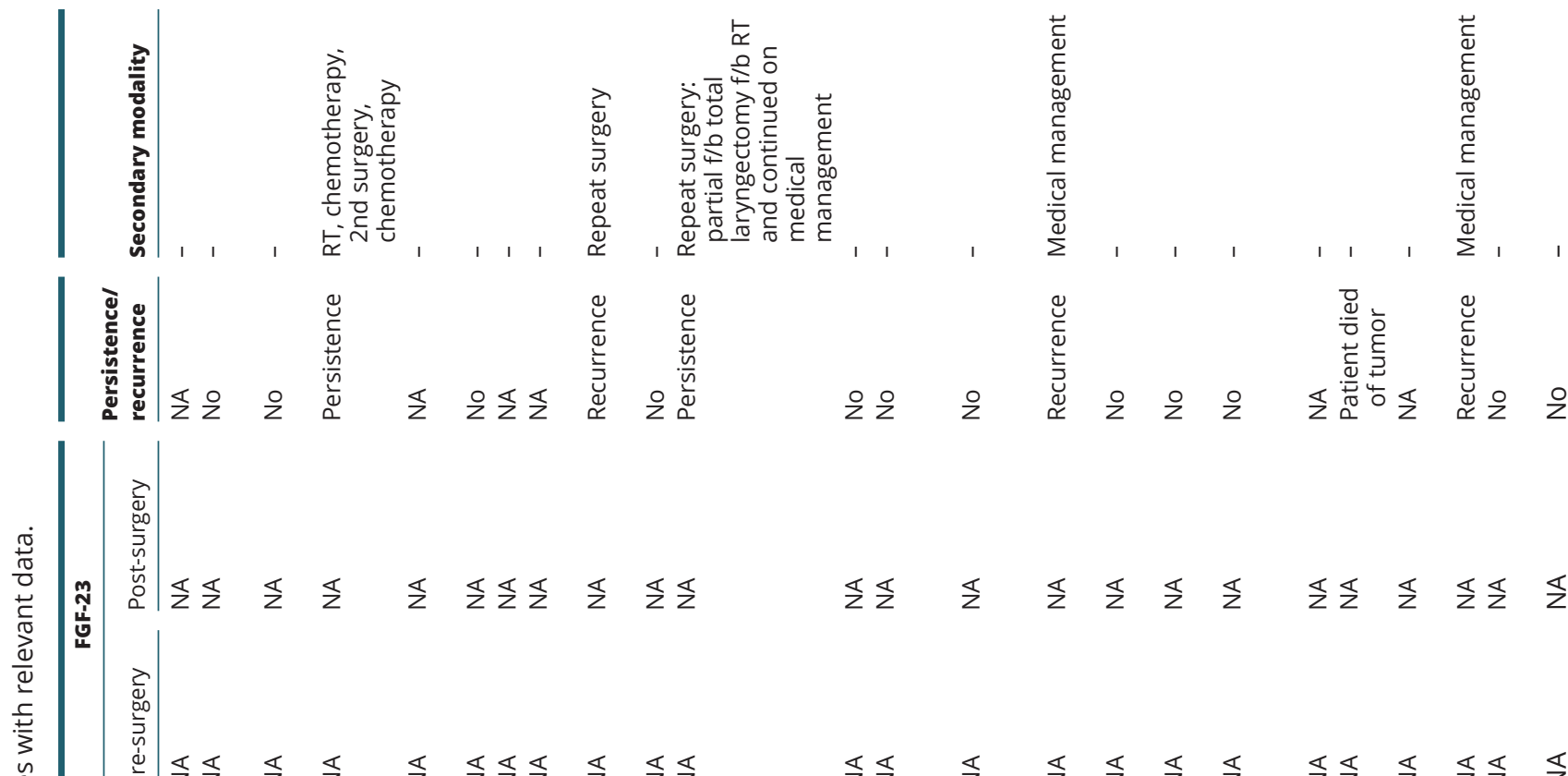

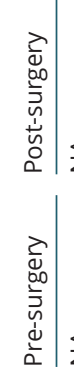

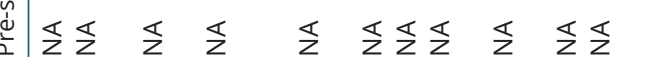
$\frac{\pi}{2} \quad \frac{1}{2}$

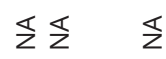

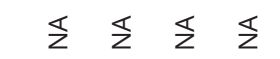

$\frac{1}{z} \frac{1}{z} \frac{\pi}{2}$

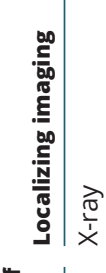

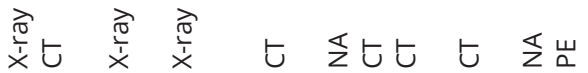

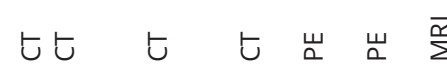

$ヒ ち \quad 七 \quad ち \bar{\Sigma}$

○气

d

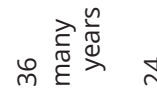

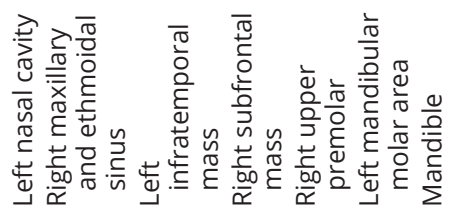

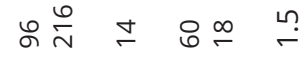

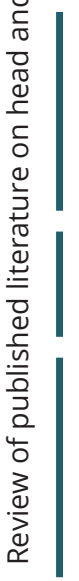

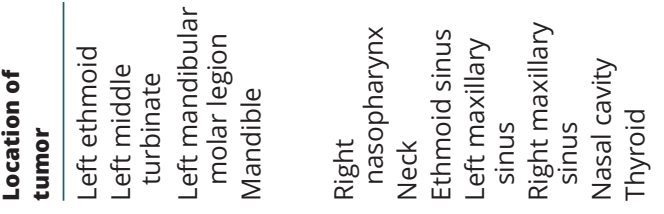

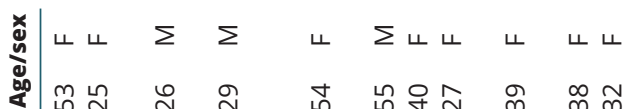

ㄴ

๖

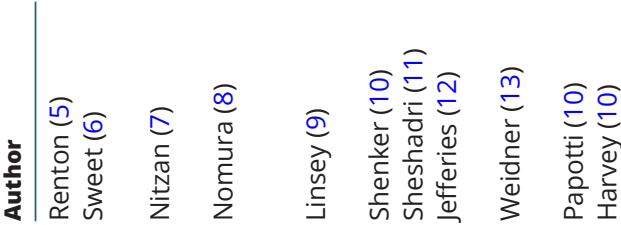

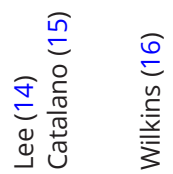

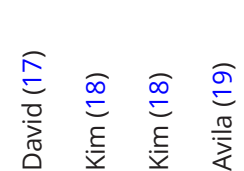

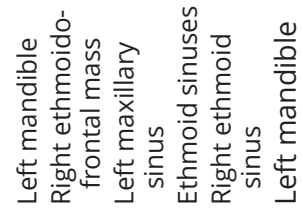

高

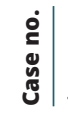

ก $0 \wedge \infty$ a 둗 


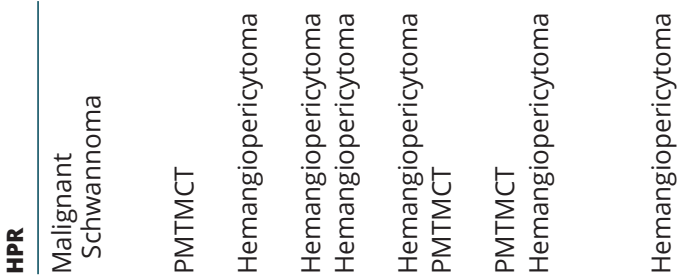
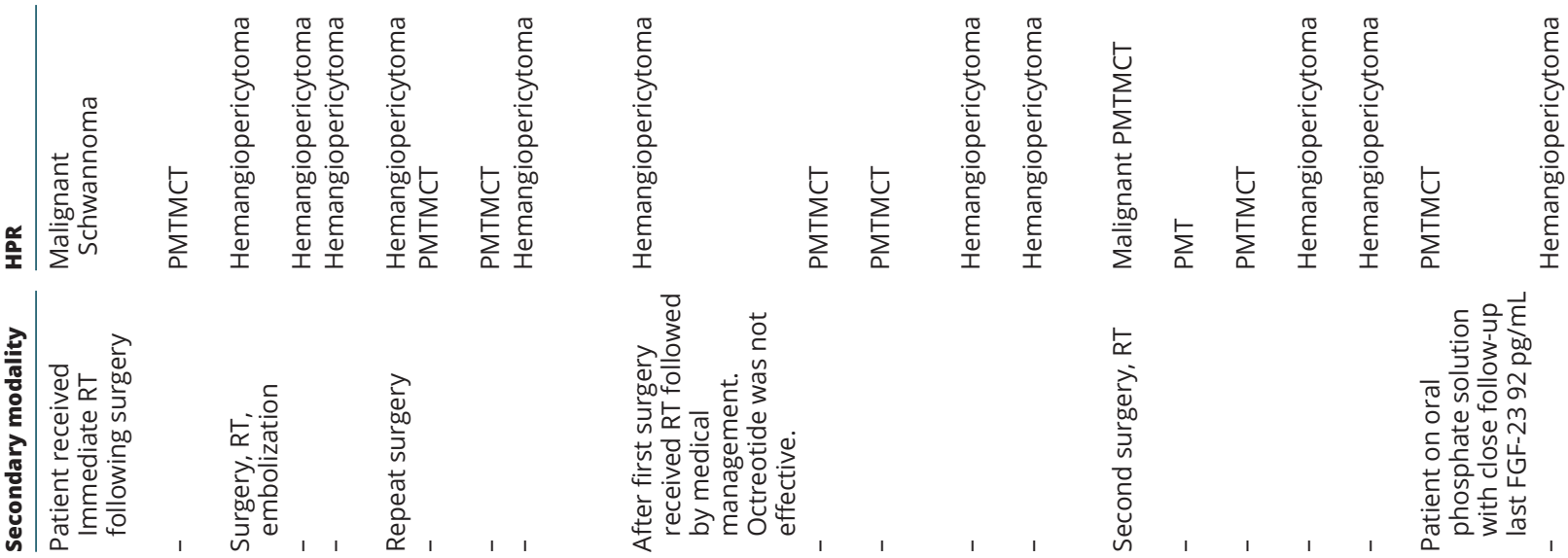

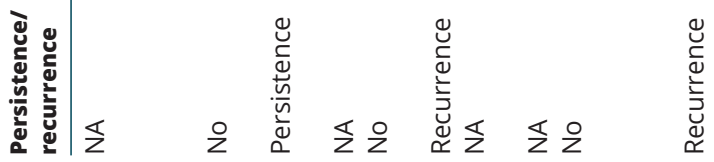

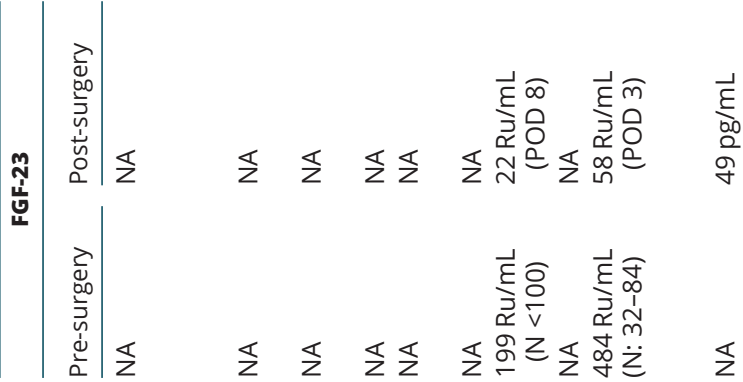
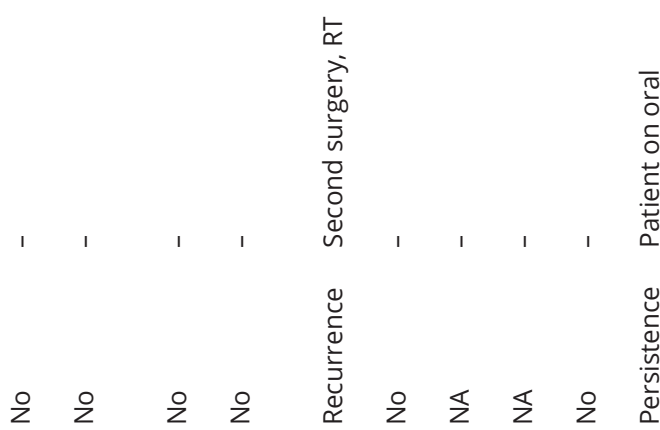

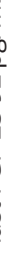

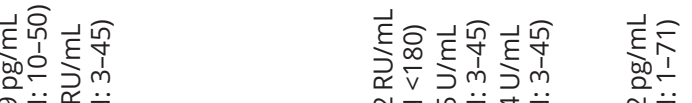

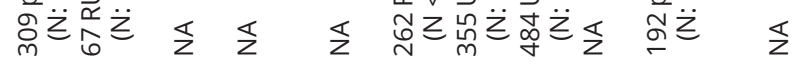

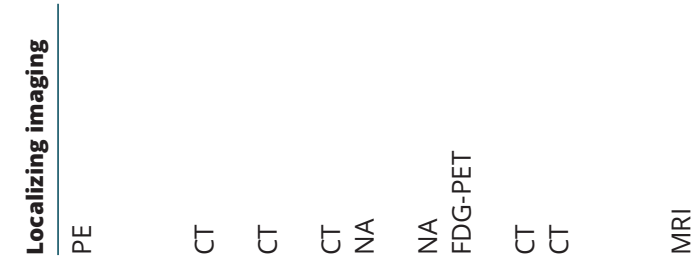

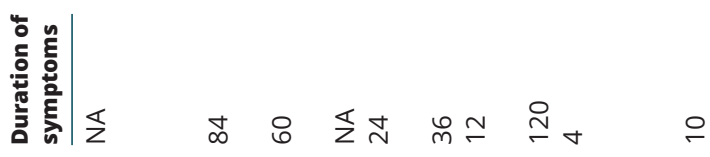

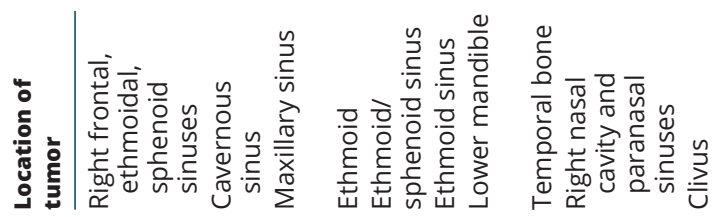

m

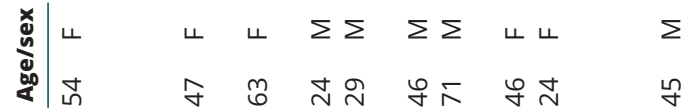

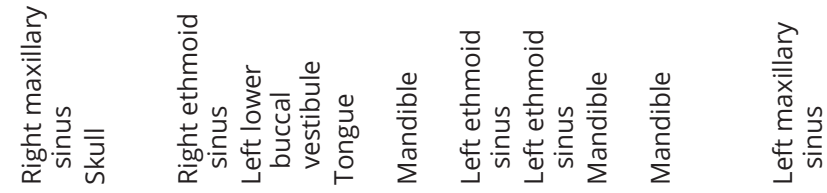

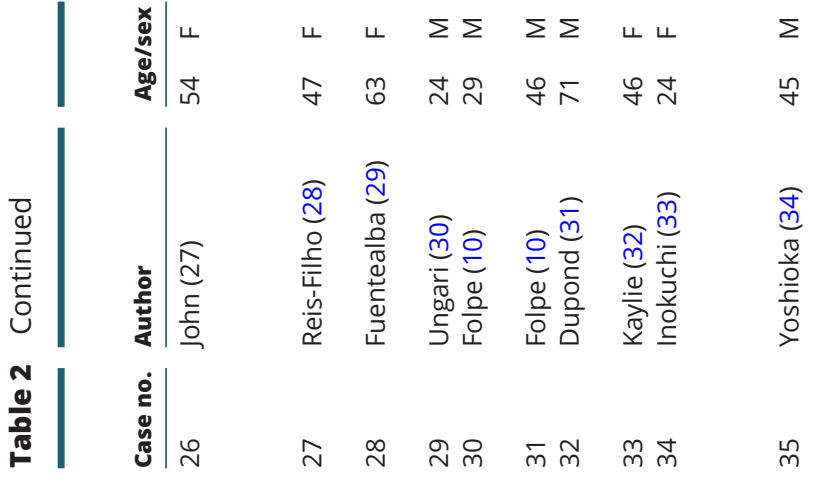 ч

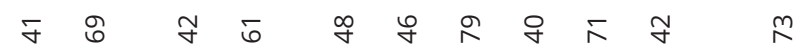




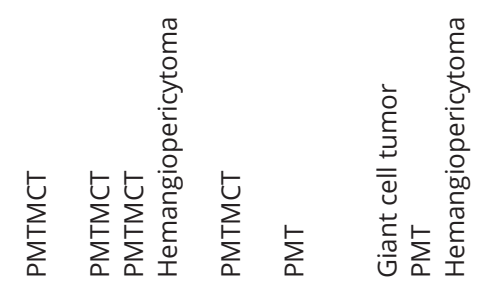

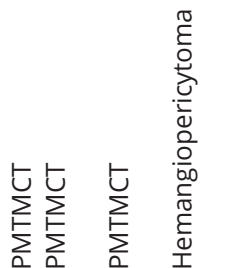

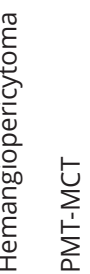
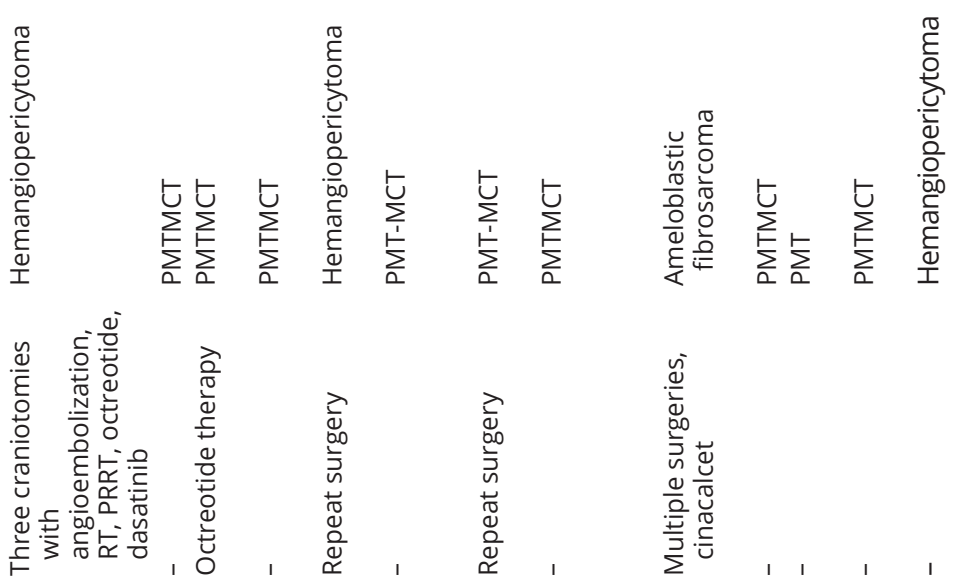

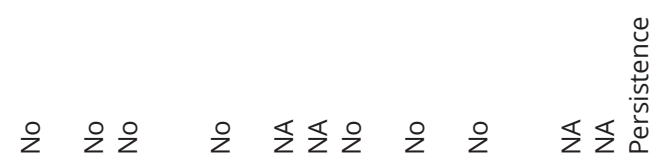

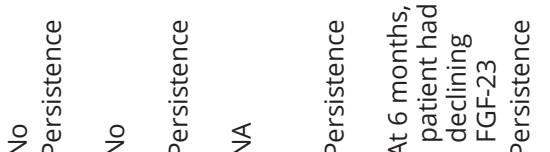

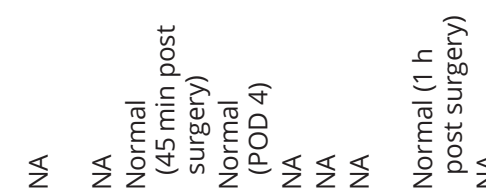

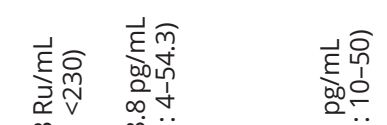

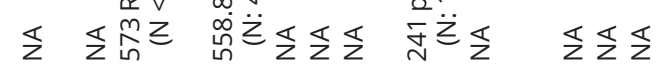

\&文$$
z a
$$

울을 운

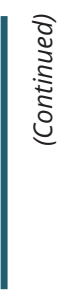

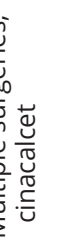

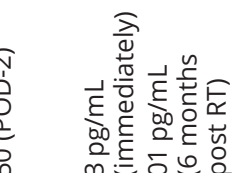

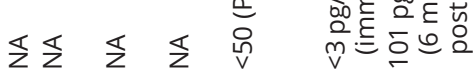

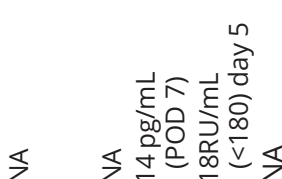

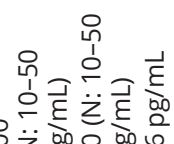

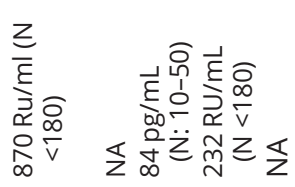

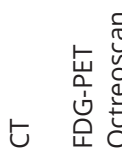

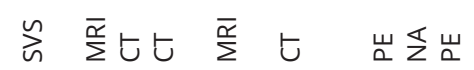

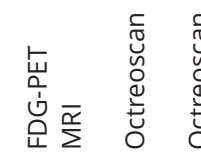

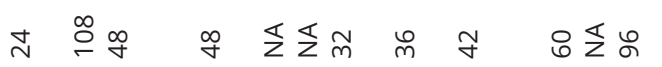

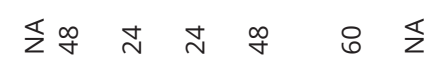

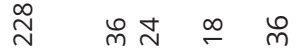

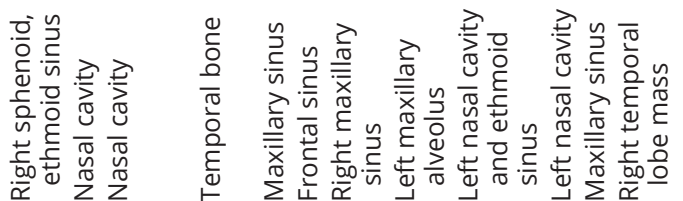

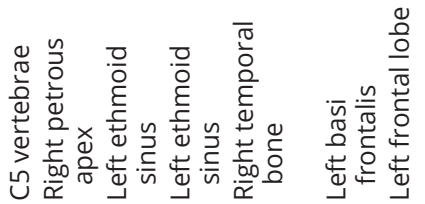

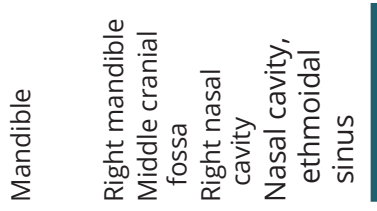

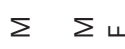

$\sqcup \quad \sqcup \Sigma \leftarrow \quad \Sigma \leftarrow$

ч $\Sigma$

$\Sigma \leftarrow$ ч $\Sigma$ レ

$\sum \sum \Sigma \Sigma L$

ถูกิธก กี

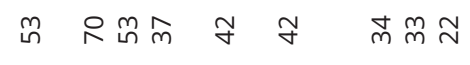

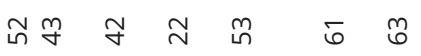

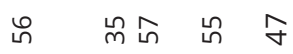

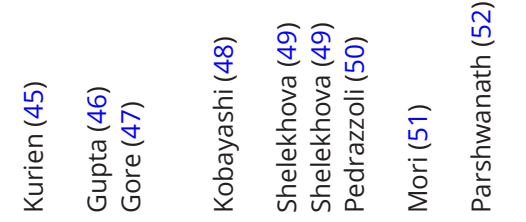

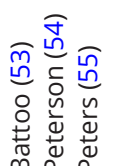

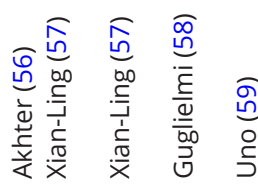

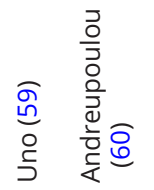

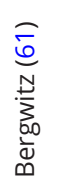

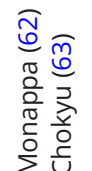

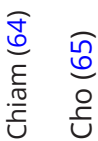

ํํ ถิ ถึ

ஸึ่

ㄴํㅇํㅇ 


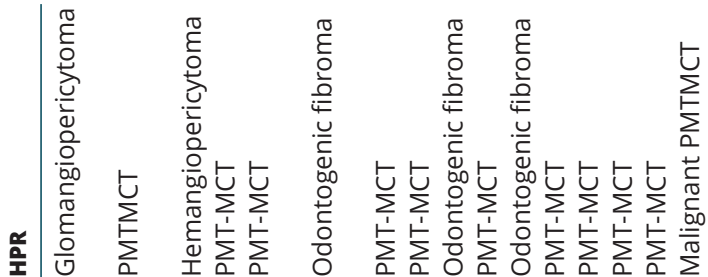

$\sum_{a}^{E}$

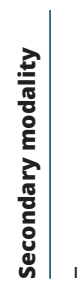

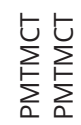
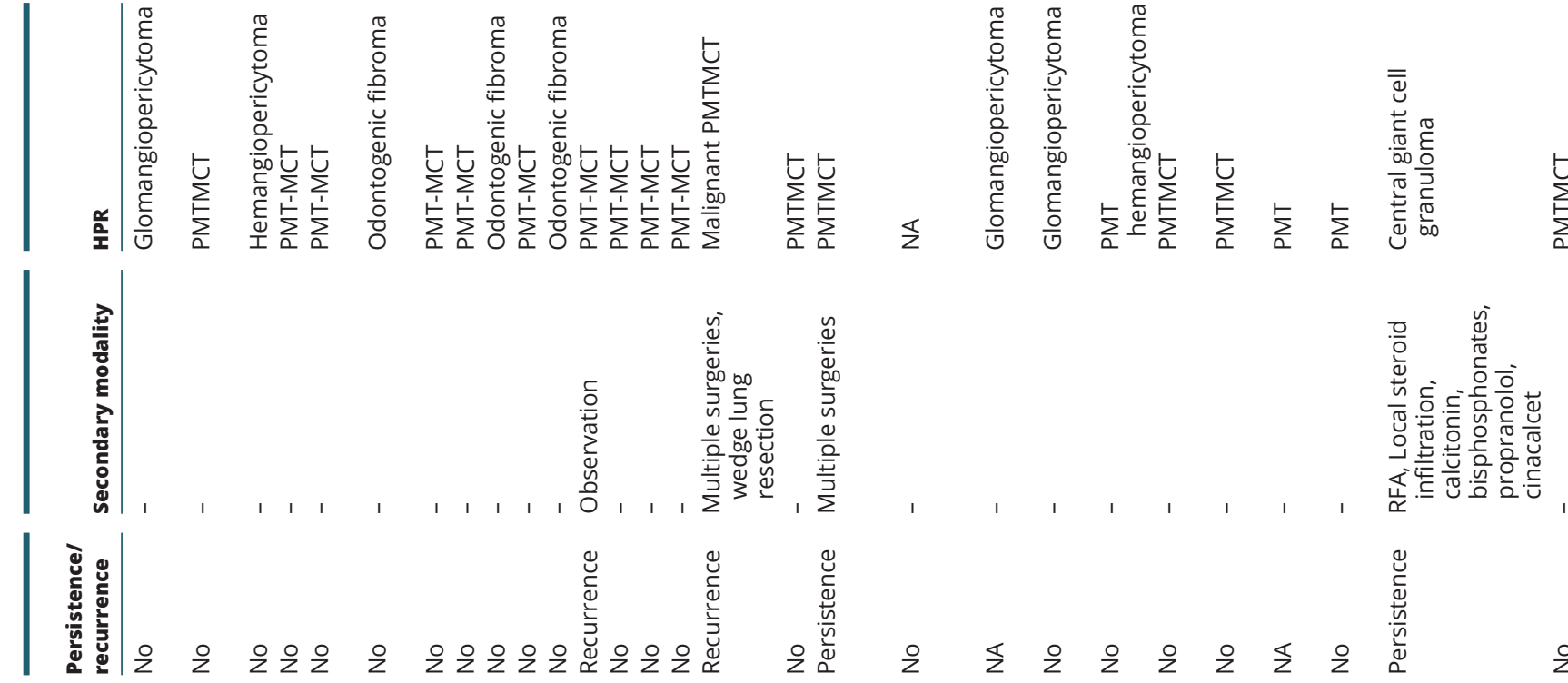

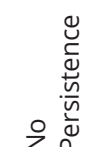

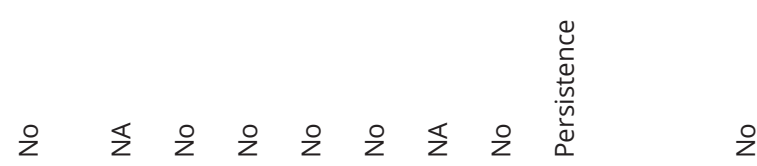

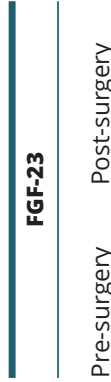

$\varangle \quad \frac{\bar{c}}{\sum^{\circ}}$

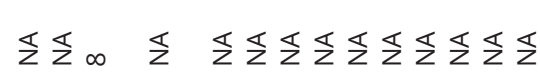

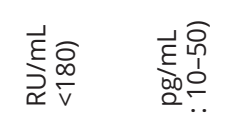

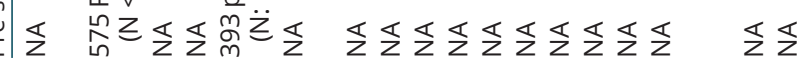

3

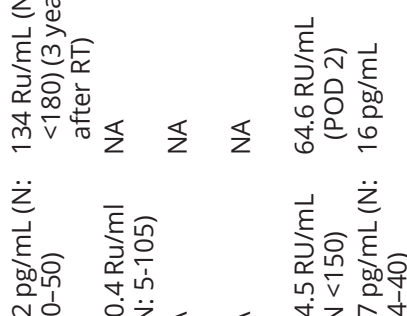

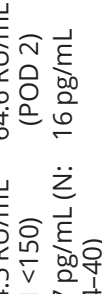

$\stackrel{0}{2}$

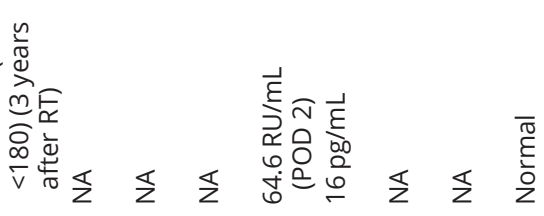

$\S$

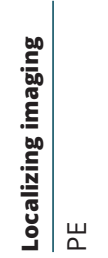

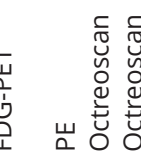

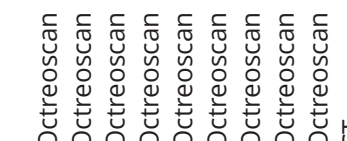

焉

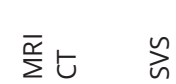
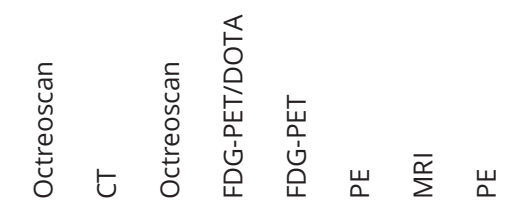

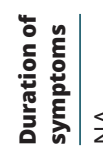

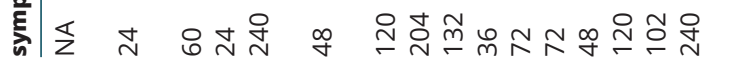

$\stackrel{m}{\infty} \stackrel{+}{\square} \approx$

$\stackrel{\infty}{\circ}$

$\stackrel{i}{m}$

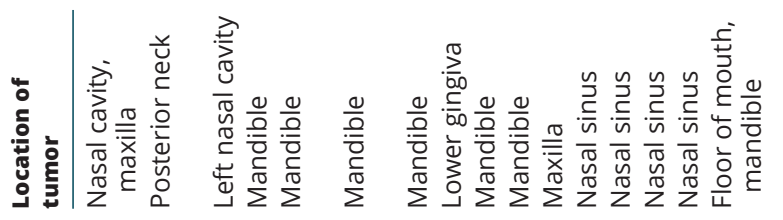

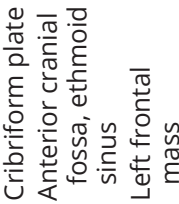

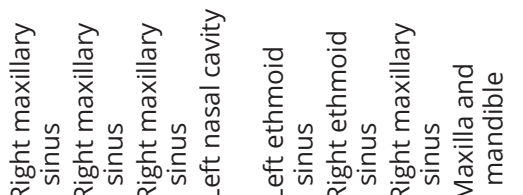

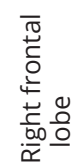

崖

$\leftarrow \Sigma$

4

遇

$\stackrel{\infty}{\sim} \underset{m}{\infty}$

$\sum$ ᄂ $\Sigma \Sigma \leftarrow \Sigma \Sigma$

ㅇ ㅇ g $\stackrel{m}{m}$ i $\stackrel{\infty}{m}$

守

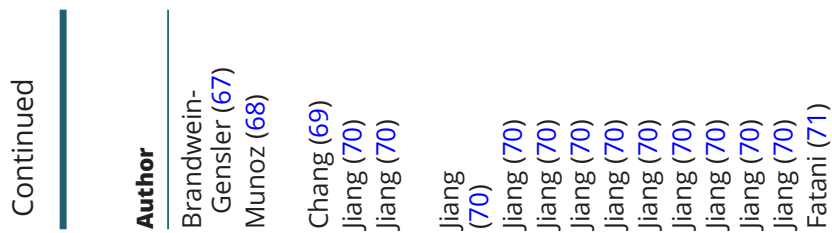

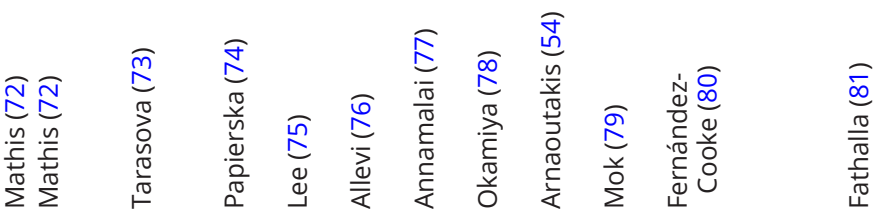

苋

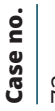

ハ

$\infty$

ㅇ

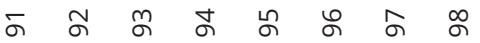

\% 


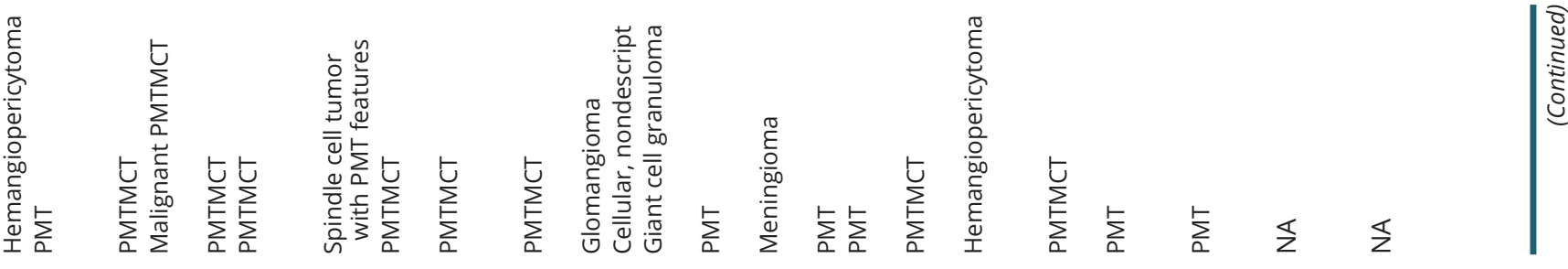

CONNECTIONS

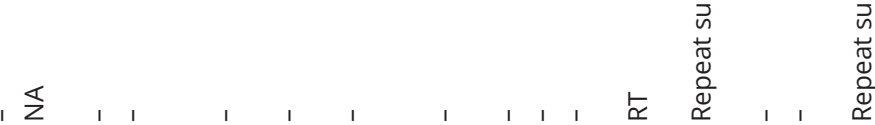

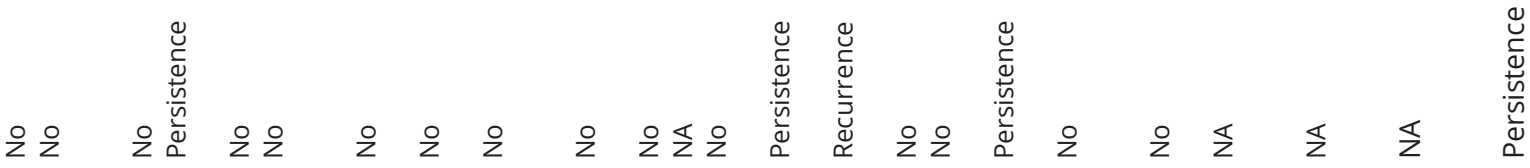

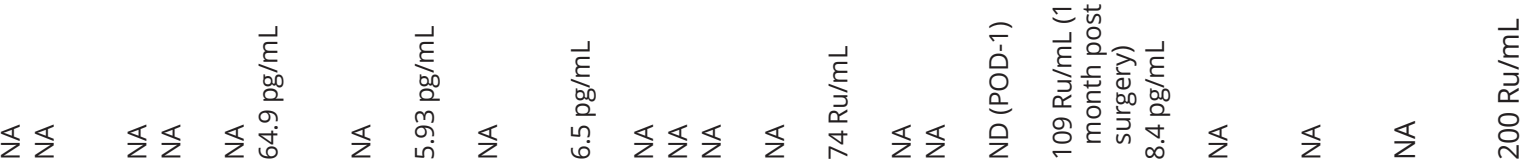

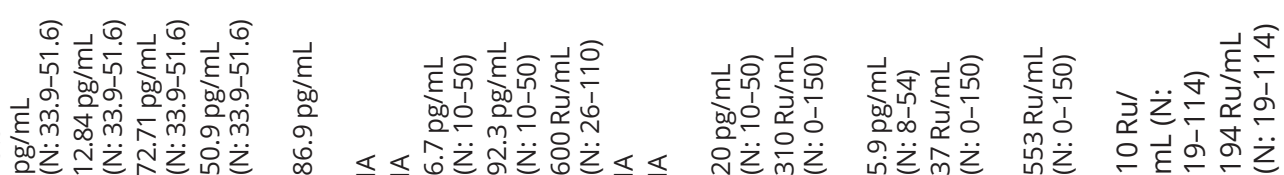

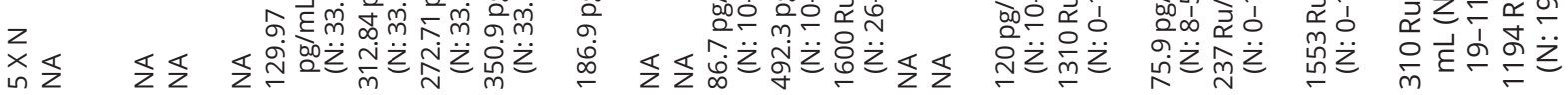

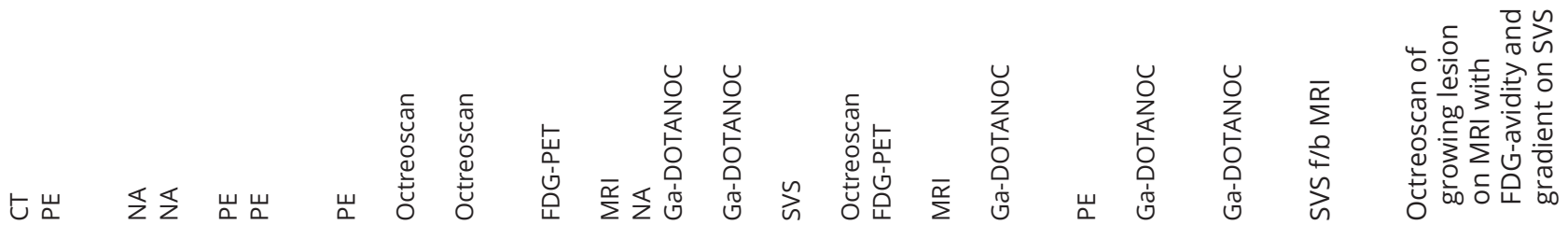

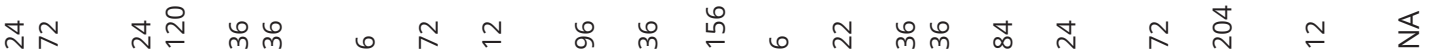

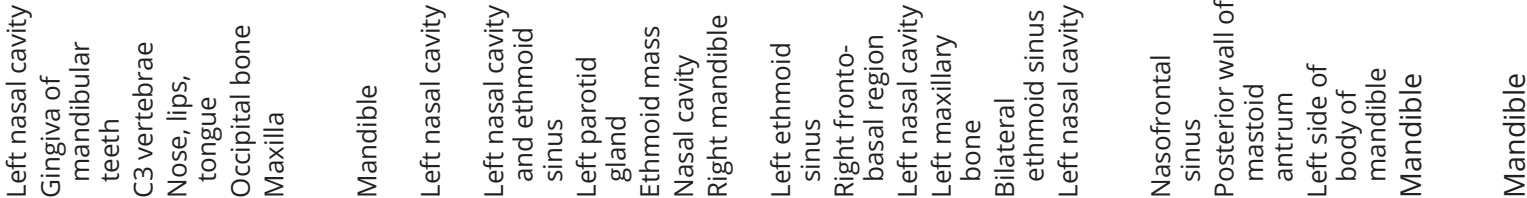

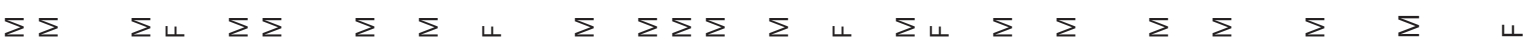

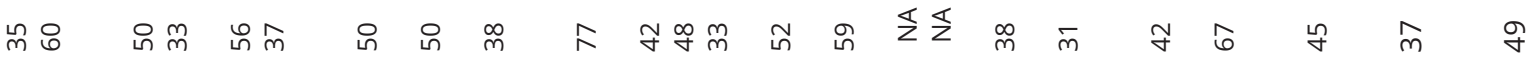

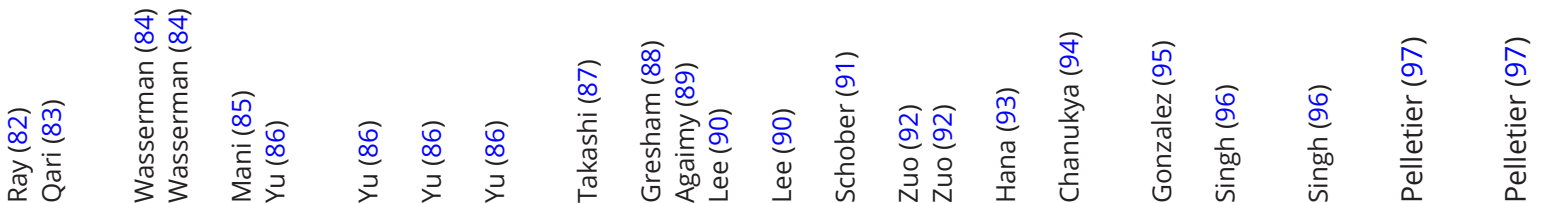

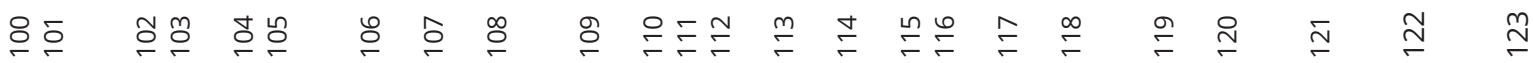




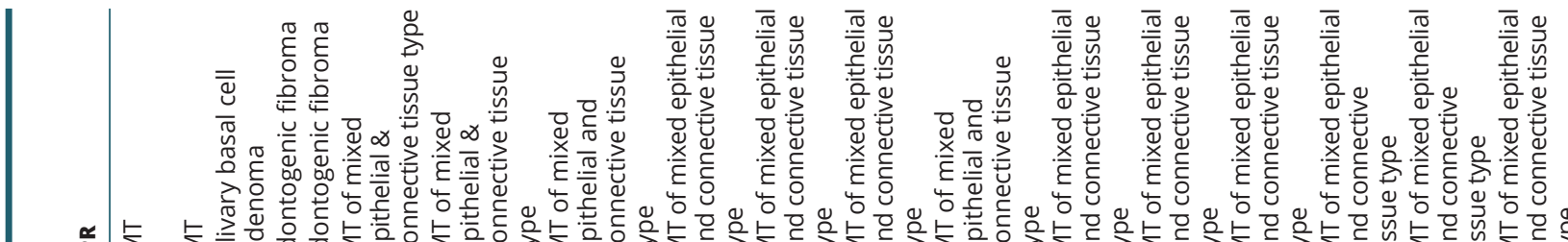

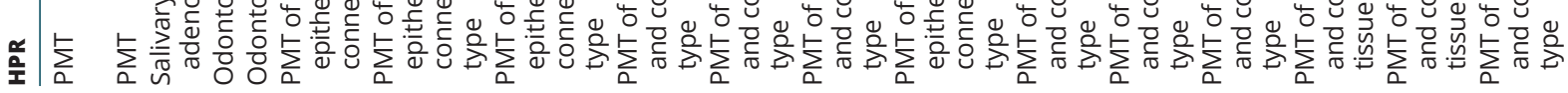

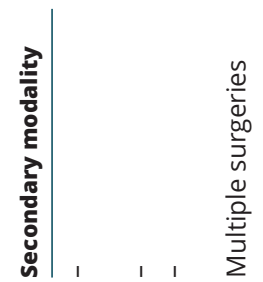

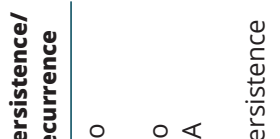

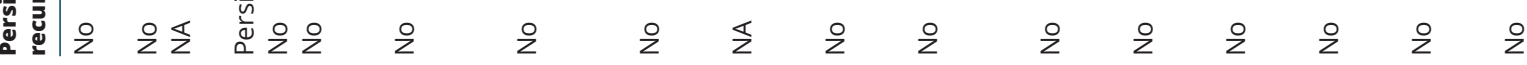
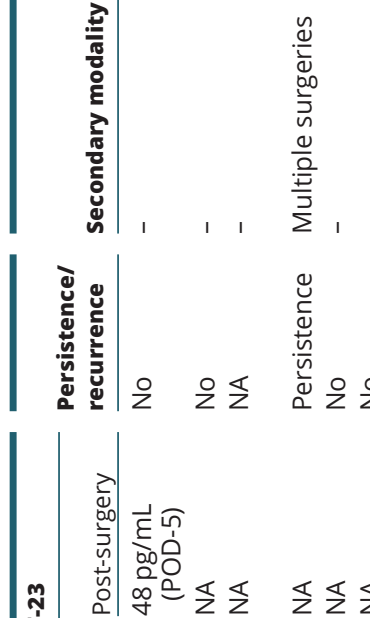

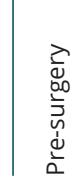

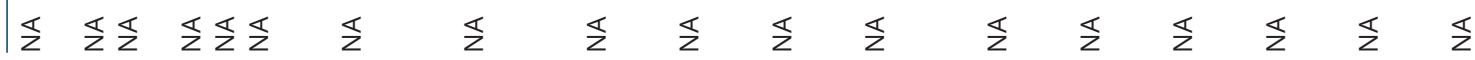

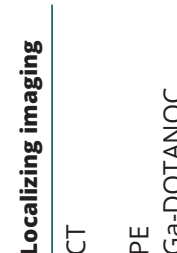

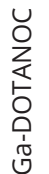

竞

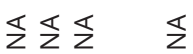

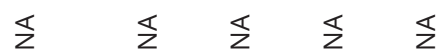

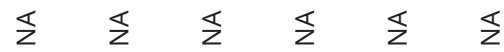

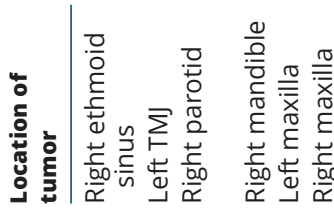

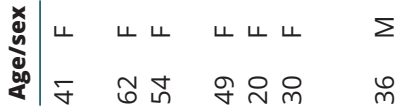

$\sqrt{n}$

+ $8 x$



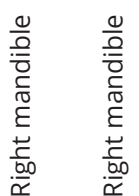

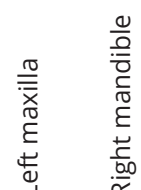

$\stackrel{\infty}{\circ}$

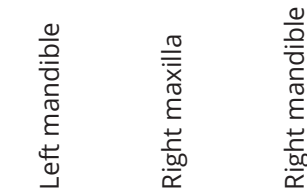

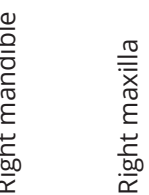

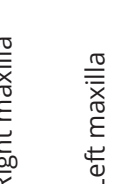

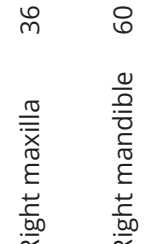

$\stackrel{\sim}{i}$

ก

$\stackrel{\sim}{\leftarrow} \stackrel{\text { ㅇ }}{\text { ก }}$

$\because \quad$

空

\section{ชิ}

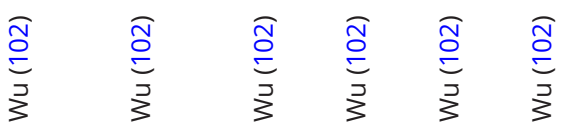

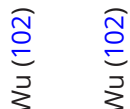

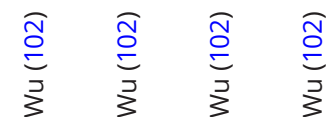




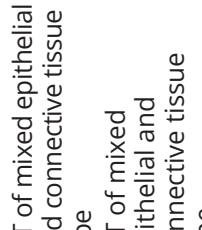

CONNECTIONS

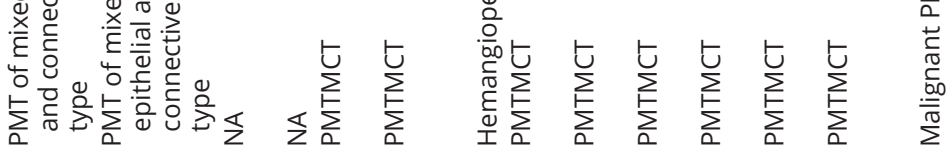
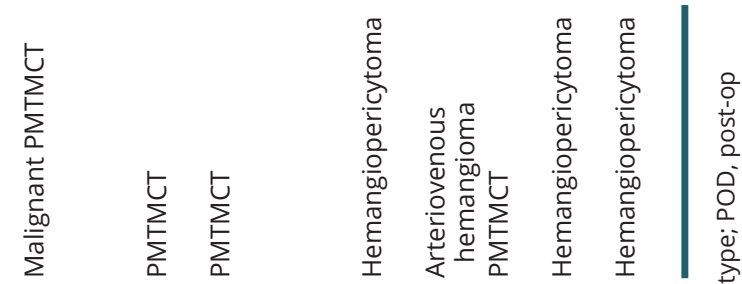

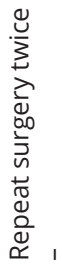

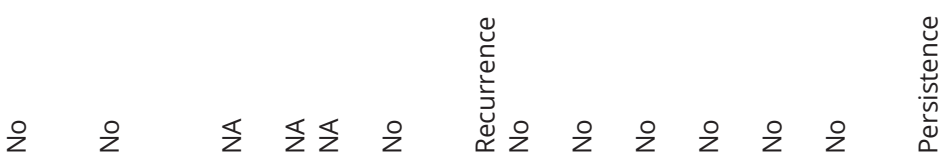

เ
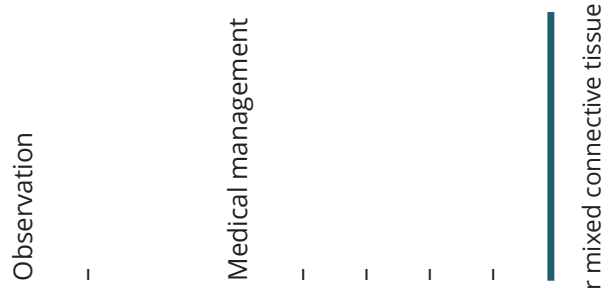

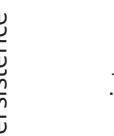

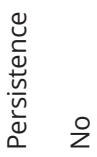

$\frac{}{\frac{\pi}{\pi}}$

울 운

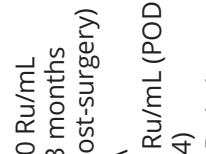

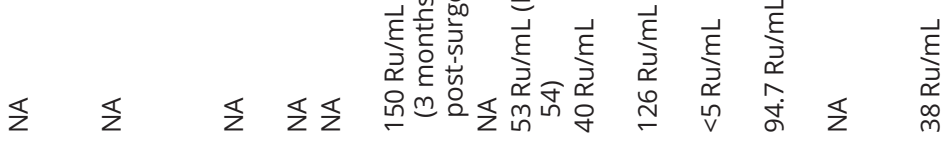

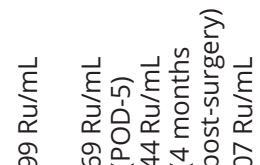

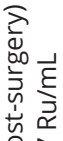

命

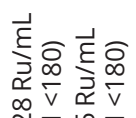

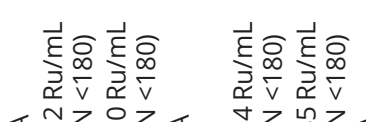

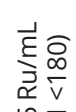

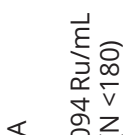

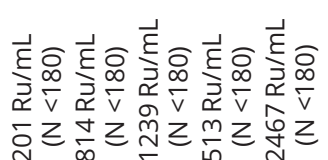

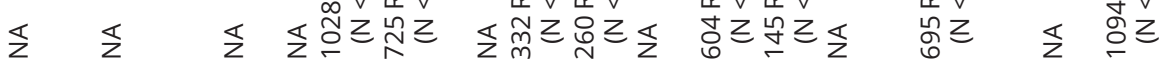

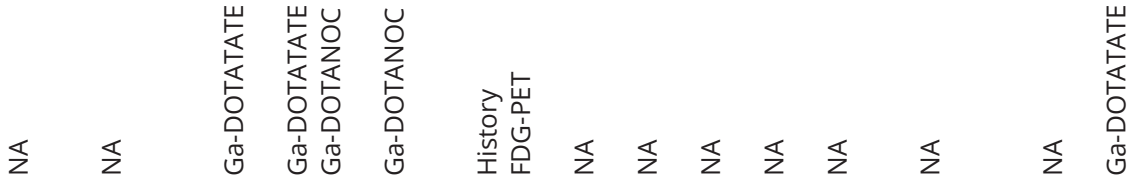

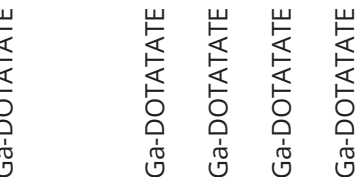

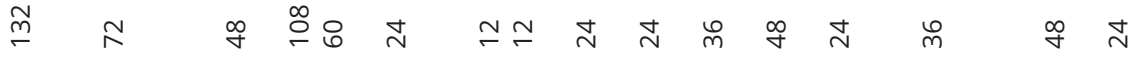

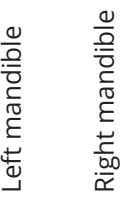

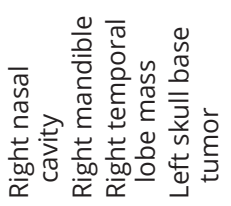

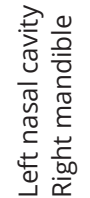

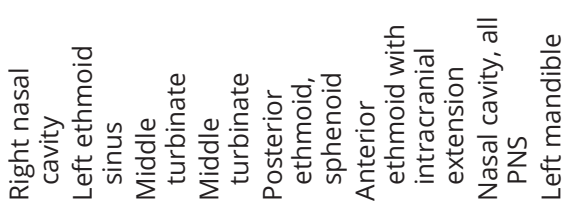

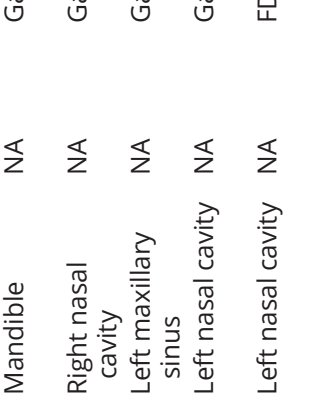

$\Sigma \quad \Sigma$

ч $\Sigma \sum$ แ

$\sqcup \Sigma$ н $\Sigma \Sigma \Sigma \Sigma$

४ ᄂ

in $\stackrel{n}{n}$

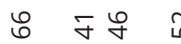

워

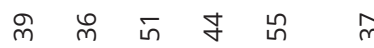

กิ

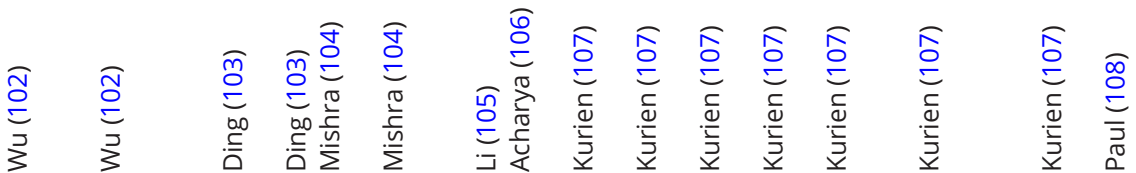

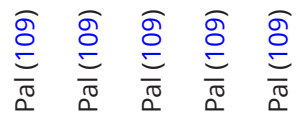

$\begin{array}{lllll}\Sigma & \llcorner & \llcorner & \Sigma & \llcorner \\ \stackrel{\infty}{N} & \sim & \infty & \infty & \infty\end{array}$

。

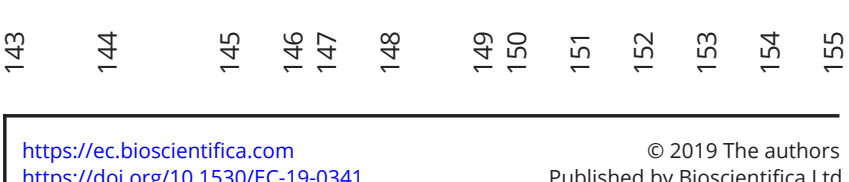


Table 3 Summary of literature review.

\begin{tabular}{|c|}
\hline Parameter \\
\hline Age (years) (mean \pm s.D.) \\
\hline Sex \\
\hline Location of tumor \% (no.) \\
\hline Paranasal sinuses \\
\hline Mandible \\
\hline Intracranial \\
\hline Maxilla \\
\hline Oral cavity \\
\hline Skull \\
\hline Parotid \\
\hline Posterior neck \\
\hline Cervical vertebra \\
\hline Infratemporal fossa \\
\hline Mastoid antrum \\
\hline Thyroid \\
\hline Local symptoms \% (no.) \\
\hline Hypophosphatemic symptoms \\
\hline Muscle weakness \% (no.) \\
\hline Fractures \% (no.) \\
\hline Bone pains \% (no.) \\
\hline Bony deformities \% (no.) \\
\hline Duration of symptoms (months), median (IQR) \\
\hline Biochemical profile \\
\hline S. Calcium (mg \%) (mean \pm S.D.) \\
\hline S. Phosphorus (mg \%) (mean \pm S.D.) \\
\hline Pre-op \\
\hline Post-op \\
\hline S. Alkaline phosphatase (U/L) (median (IQR)) \\
\hline TMP/GFR (median (IQR)) \\
\hline TRP (median (IQR)) \\
\hline PTH (pg/mL) (median (IQR)) \\
\hline $1,25(\mathrm{OH}) 2$ vitamin D3 (pg/mL) (median (IQR)) \\
\hline FGF-23 (Pre-op) (median (IQR)) \\
\hline X ULN \\
\hline C-terminal (Ru/mL) \\
\hline Intact (pg/mL) \\
\hline FGF-23 (Post-op) \\
\hline C-terminal (Ru/mL) \\
\hline Intact $(\mathrm{pg} / \mathrm{mL})$ \\
\hline Tumor size (cm) (median (IQR)) \\
\hline Localization imaging \% (no.) \\
\hline History and PE \\
\hline X-ray \\
\hline CT scan \\
\hline MRI \\
\hline Octreotide scintigraphy \\
\hline FDG-PET/CT \\
\hline Ga-DOTA-based PET/CT \\
\hline Selective venous sampling of FGF-23 \\
\hline Primary modality of treatment \% (no.) \\
\hline Surgery \\
\hline Radiation therapy \\
\hline Combined surgery + radiation therapy \\
\hline Complete response to primary treatment \% (no.) \\
\hline Persistent disease \% (no.) \\
\hline Follow-up (months) \\
\hline Recurrence \% (no.) \\
\hline Time to recurrence (months) (range) \\
\hline
\end{tabular}

\begin{tabular}{c}
\hline Value \\
\hline $46 \pm 14$ \\
$81: 81$ \\
$43.7(76)$ \\
$21.5(34)$ \\
$11.8(19)$ \\
$9(13)$ \\
$6.2(10)$ \\
$1.2(2)$ \\
$1.3(2)$ \\
$1.3(2)$ \\
$1.3(2)$ \\
$0.7(1)$ \\
$0.7(1)$ \\
$0.7(1)$ \\
$44.1(49)$
\end{tabular}

No. of patients with available data

$77.9(106)$ 
Table 3 Continued

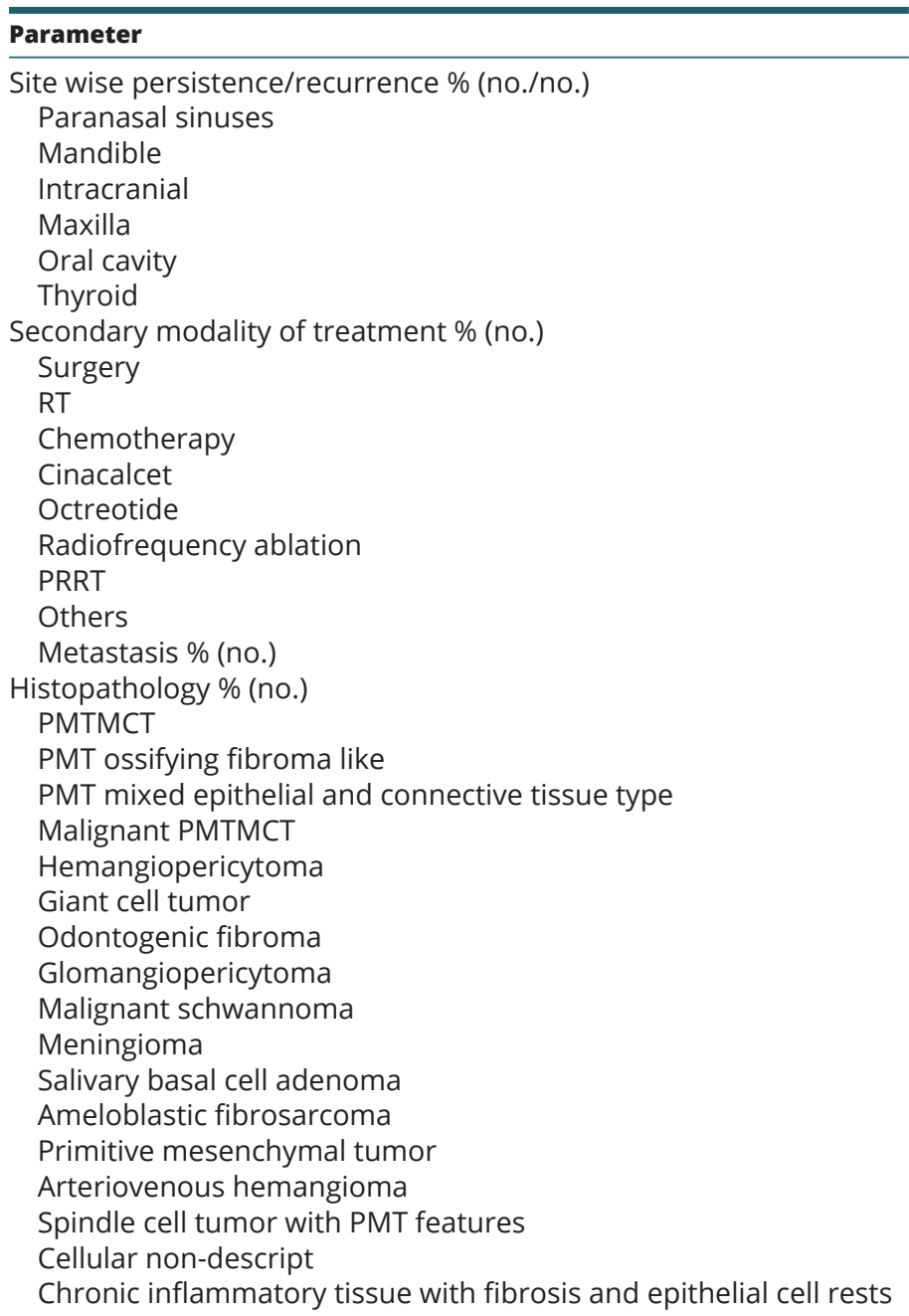

\begin{tabular}{cc}
\hline Value & \\
\cline { 1 - 2 } $14.4(7 / 4)$ & 76 \\
$17.6(6 / 0)$ & 34 \\
$36.8(4 / 3)$ & 19 \\
$7.6(1 / 0)$ & 13 \\
$33.3(1 / 2)$ & 10 \\
$100(1)$ & 1 \\
& 26 \\
$65.4(17)$ & \\
$30.8(8)$ & \\
$7.7(2)$ & \\
$7.7(2)$ & \\
$7.7(2)$ & \\
$3.8(1)$ & \\
$3.8(1)$ & \\
$3.8(1)$ & \\
$2.7(4)$ & \\
$48.7(77)$ & \\
$1.3(2)$ & \\
$9.5(15)$ & \\
$3.2(5)$ & \\
$22.8(36)$ & \\
$3.2(5)$ & \\
$3.2(5)$ & \\
$2.5(4)$ & \\
$0.6(1)$ & \\
$0.6(1)$ & \\
$0.6(1)$ & \\
$0.6(1)$ & \\
$0.6(1)$ & \\
$0.6(1)$ & \\
$0.6(1)$ & \\
$0.6(1)$ & \\
$0.6(1)$ & \\
& \\
\hline
\end{tabular}

Complete surgical removal with wide margin of excision remains the cornerstone of management in these cases (3). This is particularly difficult in intracranial tumors resulting in persistent disease as noted in both our patients with intracranial tumors.

S. Phosphorus and FGF-23 levels are used for postoperative surveillance. Half-life of FGF-23 is very short and one can document it immediately post-operatively (93). Persistent elevation of FGF-23 was noted post-operatively in two patients (cases 1 and 6), which normalized on re-evaluation after 3 months. This observation has been previously reported particularly with C-terminal FGF-23 assay $(108,109)$. Phosphate supplements are discontinued post-operatively to allow for surveillance. Reimaging is performed in patients with persistent symptoms and biochemically active disease.

In recurrent or persistent cases, complete tumor removal resulted in cure in two patients, hence, this remains the preferred approach at our institute. In inoperable cases, two patients received external beam radiotherapy (EBRT) and one patient received peptide receptor radiotherapy (PRRT). In one patient (Case 2) EBRT was given after first surgery due to difficult tumor location at petrous apex. He had a gradual and complete response to RT over next 4 years. In another scenario (case 3), the patient had persistent disease after functional endoscopic sinus surgery (FESS) for left ethmoid sinus tumor. Following two repeat FESS, patient was considered for EBRT for persistent disease. Patient received IMRT 54Gy in 30 fractions. S. Phosphorus and FGF-23 normalized gradually over one and half years and this patient who was previously bedbound is now walking without any support.

One patient (case 5) in our cohort has received PRRT for persistent disease after two surgeries for base of skull tumor (113). As tumor was Ga-DOTATATE avid having

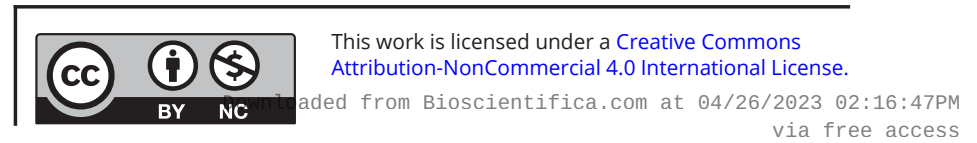


Krenning score IV, patient was considered for PRRT after a thorough discussion in a multidisciplinary meeting. This patient has stable disease after two cycles of PRRT with 150-200 uCi ${ }^{177} \mathrm{Lu}$-DOTATATE.

PMTMCT remains the commonest histopathologic entity in these patients. We also reported one patient for each of the following: PMT-OF like, odontogenic fibroma and hemangiopericytoma in our cohort. Detailed histopathological findings for cases three, four and six have been published previously (114).

Although the sample size of cohort 1 was small, the epidemiological data are similar to cohort 2 . There is an increased prevalence of local symptoms at presentation and higher rate of persistence following primary surgery at our center. This could be attributed to referral bias to a tertiary care center.

\section{Cohort 2}

Here we present a detailed review of published English literature for TIO cases involving head and neck region $(n=163)(5,6,7,8,9,10,11,12,13,14,15,16,17,18,19$, $20,21,22,23,24,25,26,27,28,29,30,31,32,33,34,35$, $36,37,38,39,40,41,42,43,44,45,46,47,48,49,50,51$, $52,53,54,55,56,57,58,59,60,61,62,63,64,65,66,67$, $68,69,70,71,72,73,74,75,76,77,78,79,80,81,82,83$, $84,85,86,87,88,89,90,91,92,93,94,95,96,97,98,99$, $100,101,102,103,104,105,106,107,108,109)$. This is the largest series of its kind published to date.

\section{Epidemiology}

As is the case with overall TIO literature, almost equal male:female ratio is reported in head and neck TIO patients (3). Middle age is the most common age at presentation and three pediatric cases are reported so far. TIO is a difficult diagnosis in pediatric patients as heritable hypophosphatemic rickets is a more likely diagnosis unless the tumor is evident. Fernández-Cooke et al. have reported a 3-year-old child with rickets and a jaw tumor. Two years went by before a link was established between the two and a diagnosis of TIO was made (80). In the case described by Reyes-Mugica et al. the heightened awareness of pediatric endocrinologist for this condition led to early screening with imaging and subsequent surgical removal resulting in cure within 6 weeks of onset of symptoms (25). In the third case reported by Wu et al. also the duration of hypophosphatemic symptoms was 2 years (102).

The time from symptom onset to final diagnosis remains dreadfully long. In this series of cases of TIO involving head and neck region, only 10\% $(n=14)$ were diagnosed in the first year of disease onset with majority of them having local symptoms at presentation. Feng et al. observed a misdiagnosis rate of $95.1 \%$ with 240 case-times of misdiagnoses among 144 cases of TIO even in the presence of evident hypophosphatemia in $43.1 \%$ cases (115). Reasons cited for misdiagnosis were disease rarity, insidious onset, nonspecific clinical manifestations and poor recognition by the clinician. Presence of local compressive symptoms and/or swelling in approximately $50 \%$ patients in this review highlights the problem of delayed or missed diagnosis as musculoskeletal symptoms are ignored until presentation with advanced local symptoms.

\section{Biochemical profile}

The typical biochemical profile in TIO is straightforward: hypophosphatemia with normocalcemia, moderately elevated ALP, normal PTH, inappropriately normal-tohigh urinary phosphate excretion, low serum 1,25 (OH)2 vitamin D3 and elevated FGF-23 levels (3).

FGF-23 is useful as a tumor marker. Based on two case reports, half-life of FGF-23 is between 20-50 min $(116,117)$. More recently, Hana et al. reported half-life of FGF-23 to be $18.5 \mathrm{~min}$ in a patient with intracranial PMTMCT using intact FGF-23 assay (93). This allows FGF-23 to be used for intraoperative monitoring to determine the extent of tumor removal. Immediate post-op decline in FGF-23 levels within normal range is reported by other investigators $(36,47,51,59)$ as well. Elston et al. reported discordant increase in C-terminal FGF-23 post-op which has not been confirmed by other studies (36). As previously stated, persistent elevation in C-terminal FGF-23 in immediate post-operative period has been documented despite complete tumor removal $(108,109)$. With no reports on levels of other postulated phosphotonins like matrix extracellular phosphoglycoprotein (MEPE) and secreted frizzled-related protein 4 (SFRP4) in patients with TIO, their role still remains unclear (118).

\section{Location of tumor}

Most common site for TIO in head and neck region is paranasal sinuses. Among them, ethmoid sinuses are the most common site followed by maxillary, sphenoid and frontal sinuses. Most common tumors are PMTMCT, hemangiopericytoma and glomangiopericytoma, in descending order. The second most common site is bony tumors arising from the mandible and maxilla with

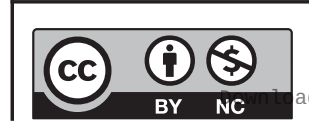

This work is licensed under a Creative Commons Attribution-NonCommercial 4.0 International License. ded from Bioscientifica.com at $04 / 26 / 2023$ 02:16:47PM 
odontogenic fibroma and, PMT of mixed connective tissue and epithelial components as special tumor types. Third position is for intracranial tumors involving anterior cranial fossa, middle cranial fossa, and posterior cranial fossa, in descending order of prevalence. Reported tumors include PMTMCT, hemangiopericytoma and meningioma. Tumors of oral cavity include gingival tumors (molar/premolar), tongue and buccal vestibule in that order of occurrence. Apart from PMTMCT (including malignant) and hemangiopericytoma, tumors from this region also include giant cell tumor and ossifying fibroma. Rarely tumors have been reported from skull, parotid glands, posterior neck, infratemporal fossa, mastoid antrum, thyroid and vertebra.

\section{Localization imaging}

Classically, history of local compressive symptoms and/or visible mass on physical examination is instrumental in diagnosing TIO even in this current era of sensitive imaging modalities. Earlier clinicians were dependent on physical examination and x-rays for diagnosing TIO. Renton et al., Nitzan et al., and Nomura et al. have localized head and neck TIO through x-rays alone $(5,7$, 8). With the introduction of CT scans (1980-2000), 60\% tumors in the head and neck region were localized with this modality. The first localization of head and neck TIO on MRI was reported by Avila et al. in 1996 using MR skeletal survey (19).

Following in vitro demonstration of somatostatin receptors (SSTRs) by Reubi et al. (119), scintigraphic studies using ${ }^{111}$ In-pentetreotide for tumor localization was published by De Beur et al. in 2002 (120). Subsequently, localization with ${ }^{99} \mathrm{mTc}-\mathrm{MIBI}$ and FDG-PET scans was reported $(121,122)$. Use of FDG-PET was limited due to poor specificity of non-receptor-based imaging, and slow-growing nature of these tumors resulting in falsenegative results (96). With improved spatial resolution, lower radiation dose and more rapid whole-body tomographic imaging of PET/CT studies in comparison to scintigraphy, ${ }^{68} \mathrm{Ga}$-DOTA-based PET/CT scans became the investigation modality of choice in TIO patients $(112,123)$. Various studies have shown superiority of ${ }^{68} \mathrm{Ga}$-DOTATATE PET/CT and ${ }^{68} \mathrm{Ga}$-DOTANOC PET/CT over FDG-PET/CT and Octreoscan for tumor localization in TIO $(110,111,112)$. The largest such study is that of 54 patients by Zhang et al. using ${ }^{68} \mathrm{Ga}$-DOTATATE PET/CT reported $100 \%$ sensitivity and $90.9 \%$ specificity in lesion detection (124). Use of positron emitter radiotracer ${ }^{68} \mathrm{Ga}$ enabling PET-based imaging along with higher affinity SSTR ligands like DOTATATE (SSTR $2>5$ ) and DOTANOC
(SSTR 2,3,5) are postulated to be responsible for enhanced sensitivity of ${ }^{68} \mathrm{Ga}$-DOTA-based PET/CT over Octreoscan (112). Thereafter, Singh et al. highlighted the issue of multiple low-grade benign uptakes using ${ }^{68} \mathrm{Ga}$-DOTANOC PET/CT especially at fracture sites and described the use of SUVmax and anatomical imaging showing soft tissue component in the lesion to pinpoint the causal lesion (96). In summary, Ga-DOTA-based PET/CT is superior to other functional studies like FDG-PET and Octreoscan, but its utilization will depend on local availability and expertise (119).

Selective venous sampling of FGF-23 has been studied for accurate localization of TIO. Kobayashi et al. used selective venous sampling as an initial guiding modality localizing the tumor to right head and neck region, although on retrospect distortion of right external ear canal was noted and no prior functional imaging was done to localize the tumor (48). Andreopoulou et al. reported sensitivity of $87 \%$ and specificity of $71 \%$ at FGF-23 concentration ratio of 1.6 between the venous drainage of the tumor bed and general circulation after sampling 17 major veins and their branches (60). They concluded that selective venous sampling is not useful in the absence of suspicious lesion on imaging studies and its use should be limited to cases with multiple suspicious sites or before resection in anatomically challenging cases. In 2017, Lee et al. reported contrasting results. In their cohort, five patients negative on both ${ }^{111}$ Indiumoctreotide scintigraphy and FDG-PET/CT were subjected to selective blood sampling from 10 to 14 sites (90). They identified the culprit lesion on follow-up with targeted MRI or whole-body Ga-DOTATOC in four patients. Tarasova et al. and Shober et al. have used selective venous FGF-23 sampling to confirm the SSTR expressing meningioma to be the FGF-23 secreting culprit lesion as many meningiomas are avid on SSTR-based imaging but may not be the source of FGF-23 (73). In summary, in the current era of SSTR-based imaging, the role of this modality seems to be limited to cases with multiple suspicious uptake sites, intracranial lesions consistent with meningioma, and lastly in imaging negative cases to identify a target for focused follow-up imaging.

\section{Treatment}

\section{Primary modality}

Complete surgical resection with adequate wide margin remains the treatment of choice in these tumors (3). This is supported in head and neck TIO cases where anatomical

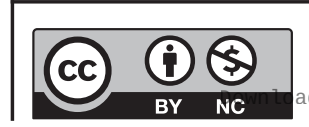

This work is licensed under a Creative Commons Attribution-NonCommercial 4.0 International License. ded from Bioscientifica.com at 04/26/2023 02:16:47PM 
sites less amenable for this approach have higher persistence or recurrence rate for example intracranial tumors. Hana et al. also reiterated this principle in their report on recurrent anterior skull base tumor with enbloc tumor removal followed by filling of the large skull base defect with pedicle subgaleal flap resulting in absence of recurrence over 25-month follow-up (93).

Stereotactic radiotherapy has been described in two cases as primary modality. Both patients had frontal lobe tumors and both refused surgery. One patient had lower plasma FGF-23 and oral phosphorous requirement at 6-month follow-up. The details of RT are not described in this case report (60). The second patient received $60 \mathrm{~Gy}$ of fractionated stereotactic radiotherapy over 5 weeks (73). On follow-up, patient was off phosphorus supplement and had normal FGF-23 concentration after 4 years. The tumor was stable with areas of multiple small hemorrhages. BMD improved by approximately $50 \%$ with no evident new fracture. As the tumors are slow growing, radiotherapy is deemed to be less effective (3).

Surgery combined with adjuvant post-op radiotherapy was used by John et al. in a case of invasive 'malignant schwannoma' (27). Over 2.5 years of follow-up, serum phosphorus normalized but $1,25(\mathrm{OH})$ vitamin D3 was persistently low. MRI showed no evidence of residual/recurrent tumor. Similarly, Lee et al. described a case where the patient received post-operative radiotherapy following incomplete removal of an ethmoid tumor, which resulted in normal serum phosphorus with no residual tumor on MRI after completion of RT (90).

In summary, although complete surgical excision remains the treatment modality of choice, in rare cases radiation therapy can be used with an expectant slow response.

\section{Persistent/recurrent disease}

Persistent/recurrent disease signifies failure of complete resection of the tumor after primary excision. This occurs more commonly in intracranial disease and oral cavity lesions where enbloc tumor removal is challenging and leads to higher surgical morbidity and complications. Serial biochemical follow-up is essential as true recurrences after complete biochemical resolution are known, but usually it is the recurrence of symptoms which brings the disease to surface.

After anatomic imaging to confirm the site of tumor recurrence, re-exploration of the surgical site along with attempted enbloc removal remains the preferred approach. Out of eleven patients with persistent/recurrent disease who have ANED on follow-up, eight have been treated with re-surgery alone.

In persistent cases multiple re-surgeries, radiotherapy, cinacalcet and octeotride have been used with limited success. Seufert et al. reported a patient with left thigh TIO localized on octreotide scinitigraphy having complete resolution of phosphaturia and normalization of serum phosphorus with 50-100 $\mu$ g of octreotide thrice a day in preoperative setting (125). However, this initial success has not been replicated in subsequent studies $(34,126)$. Extrapolating from patients with hypoparathyroidism with elevated FGF-23 and serum phosphorus levels, Gellers et al. advocated for the use of cinacalcet in the treatment of TIO (127). But development of hypercalciuria and hypocalcemia limits the use of cinacalcet in this cohort. Disease stability with dasatinib has been reported (55). As these tumors also express SSTR, PRRT remains a potentially useful option in tumors showing Krenning III/IV uptake on ${ }^{68} \mathrm{Ga}$-DOTATATE PET/CT (113). It has been more than a decade of successful utilization of two radiopeptides ${ }^{90} \mathrm{Y}$-DOTATOC and ${ }^{177} \mathrm{Lu}$-DOTATATE for treatment of advanced neuroendocrine tumors (NETs) (128). After binding to SSTR these peptides are internalized in tumor cells and the released breakdown products in lysosomes mediate radioactivity-induced local damage (128). Apart from our case, we could not find any other experience with PRRT in TIO literature. In patients with persistent disease, treatment with oral phosphate supplements and calcitriol is continued for symptomatic improvement.

\section{Metastases}

Four cases of malignant TIO in head and neck region are reported so far. Three of them originated from oral cavity and one from mandible. Uramoto et al. described a case of malignant PMTMCT involving tongue with lymph node metastases treated with two surgeries followed by radiation therapy with persistent disease on last follow-up (39). Bergwitz et al. reported a patient with ameloblastic fibrosarcoma of mandible with pulmonary and lymph node metastases (61). Patient had multiple recurrences and was managed with repeated surgeries, and lastly cinacalcet with persistent hypophosphatemia. Fatani et al. reported an interesting case of malignant PMTMCT arising from oral cavity who after 17 years of follow-up developed lung metastases which were resected in addition to multiple surgeries for primary disease (71). Patient was normophosphatemic on follow-up. The fourth case of malignant PMTMCT was reported by Wasserman et al. (84).

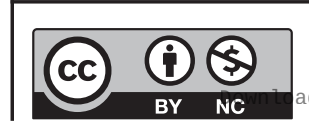


The tumor involved nose, lip and mouth. No further follow-up/management details have been reported.

\section{Histopathology}

Weidner et al. initially proposed the term phosphaturic mesenchymal tumors (PMT) and their classification into four distinct subtypes: (I) mixed connective tissue variant (MCT), (II) osteoblastoma like, (III) Non-ossifying fibroma type, (IV) ossifying fibroma like (129). In 2004, Folpe et al. reviewed all previously published cases and found that they all belong to PMTMCT category (10). In this review we have reported the revised diagnosis as mentioned by Folpe et al. In 2018, Wu et al. described a new entity called "PMT mixed epithelial and connective tissue type" which is found exclusively in alveolar bone of maxilla and mandible (102). They found this tumor to be common in males and in patients $<40$ years of age. They have proposed a revised diagnosis of previously published six cases to this new entity, but we have reported them according to the original report. Apart from PMTs, other reported tumors in head and neck region causing TIO include meningioma, salivary basal cell adenoma, malignant schwannoma, ameloblastic fibrosarcoma, and spindle cell tumor with PMT features.

\section{Study limitations}

To our knowledge this is the largest review of TIO due to tumors located in head and neck region till date. The perpatient analysis method used in this study with minute detailing of all clinically relevant published aspects is the major strength of this study. There are several limitations in this study. As the review is a retrospective analysis of published case reports, all the limitations pertaining to retrospective studies apply to it. Additionally, many case reports lacked important clinical details as majority of them focused on pathology or imaging. A meticulous attempt was made to include all published literature regarding the subject but a few studies may not have been included.

\section{Summary}

TIO in the head and neck region is a rare disorder that warrants management by a multidisciplinary team including an endocrinologist, head and neck surgeon, radiologist, nuclear physicist and pathologist. Low phosphorus with elevated FGF-23 levels in a patient with clinical features of osteomalacia and/or mass in the head and neck region should be evaluated with Ga-DOTAbased PET/CT imaging. An alternative approach would be anatomical imaging followed by biopsy in a patient with local symptoms and clinically apparent swelling. Complete surgical excision with wide margin is of utmost importance in these cases resulting in dramatic clinical and biochemical normalcy. Clinical and biochemical follow-up is necessary even after documented cure as true recurrences have been reported. Whenever complete excision is not achieved, repeat surgical excision is recommended for accessible disease burden. In inoperable cases, radiotherapy, PRRT and medical management are suitable alternatives which should be decided by a multidisciplinary team on an individual basis. Although the tumor remains benign in most cases, one must remain vigilant in case of long-standing disease due to the reported risk of metastasis. Histopathological examination in most cases reveals PMTMCT, but other types are also seen.

\section{Declaration of interest}

The authors declare that there is no conflict of interest that could be perceived as prejudicing the impartiality of the research reported.

\section{Funding}

This research has been supported by Department of Endocrinology, Seth GS Medical College \& KEM Hospital, Mumbai, India and Diamond Jubilee Society Trust (DJST), KEM Hospital, Mumbai, India.

\section{References}

1 Drezner MK. Tumor-induced osteomalacia. In Primer on Metabolic Bone Diseases and Disorders of Mineral Metabolism, 4th ed., ch 74, pp 331-337. Ed. MJ Favus. Philadelphia, PA, USA: Lippincott-Raven, 1999.

2 McCance R. Osteomalacia with Loosers nodes (Milkman's syndrome) due a raised resistance to vitamin $\mathrm{D}$ acquired about the age of 15 years. Quarterly Journal of Medicine 194716 33-46.

3 Chong WH, Molinolo AA, Chen CC \& Collins MT. Tumor-induced osteomalacia. Endocrine-Related Cancer 201118 R53-R77. (https://doi. org/10.1530/ERC-11-0006)

4 Jiang Y, Xia WB, Xing XP, Silva BC, Li M, Wang O, Zhang HB, Li F, Jing HL, Zhong DR, et al. Tumor-induced osteomalacia: an important cause of adult-onset hypophosphatemic osteomalacia in China: report of 39 cases and review of the literature. Journal of Bone and Mineral Research 201227 1967-1975. (https://doi.org/10.1002/ jbmr.1642)

5 Renton P \& Shaw DG. Hypophosphatemic osteomalacia secondary to vascular tumous of bone and soft tissue. Skeletal Radiology 19761 21-24. (https://doi.org/10.1007/BF00347723)

6 Sweet RA, Males JL, Hamstra AJ \& DeLuca HF. Vitamin D metabolite levels in oncogenic osteomalacia. Annals of Internal Medicine 198093 279-280. (https://doi.org/10.7326/0003-4819-93-2-279)

7 Nitzan DW, Marmary Y \& Azaz B. Mandibular tumor-induced muscular weakness and osteomalacia. Oral Surgery, Oral Medicine, and Oral Pathology 198152 253-256. (https://doi.org/10.1016/00304220(81)90257-7) 
8 Nomura G, Koshino Y, Morimoto H, Kida H, Nomura S \& Tamai K. Vitamin D resistant hypophosphatemic osteomalacia associated with osteosarcoma of the mandible: report of a case. Japanese Journal of Medicine 198221 35-39. (https://doi.org/10.2169/ internalmedicine1962.21.35)

9 Linsey M, Smith W, Yamauchi H \& Bernstein L. Nasopharyngeal angiofibroma presenting as adult osteomalacia: case report and review of the literature. Laryngoscope 198393 1328-1331. (https:// doi.org/10.1002/lary.1983.93.10.1328)

10 Folpe AL, Fanburg-Smith JC, Billings SD, Bisceglia M, Bertoni F, Cho JY, Econs MJ, Inwards CY, Jan de Beur SM, Mentzel T, et al. Most osteomalacia-associated mesenchymal tumors are a single histopathologic entity: an analysis of 32 cases and a comprehensive review of the literature. American Journal of Surgical Pathology 200428 1-30. (https://doi.org/10.1097/00000478-200401000-00001)

11 Seshadri MS, Cornish CJ, Mason RS \& Posen S. Parathyroid hormone like bioactivity in tumours from patients with oncogenic osteomalacia. Clinical Endocrinology 198523 689-697. (https://doi. $\operatorname{org} / 10.1111 /$ j.1365-2265.1985.tb01130.x)

12 Jefferis AF, Taylor PCA \& Walsh-Waring GP. Tumour associated hypophosphatemic osteomalacia occurring in a patient with an odontogenic tumour of the maxilla. Journal of Laryngology and Otology 198599 1011-1017. (https://doi.org/10.1017/s0022215100098091)

13 Weidner N, bar RS, Weiss D \& Strottmann MP. Neoplastic pathology of oncogenic osteomalacia/rickets. Cancer $1985 \mathbf{5 5} 1691-1705$. (https://doi.org/10.1002/1097-0142(19850415)55:8<1691::aidcncr2820550814>3.0.co;2-s)

14 Lee HK, Sung WW, Solodnik P \& Shimshi M. Bone scan in tumourinduced osteomalacia. Journal of Nuclear Medicine 199536 247-249.

15 Catalano PJ, Brandwein M, Shah DK, Urken ML, Lawson W \& Biller HF. Sinonasal hemangiopericytomas: a clinicopathologic and immunohistochemical study of seven cases. Head and Neck 199618 42-53. (https://doi.org/10.1002/(SICI)10970347(199601/02)18:1<42::AID-HED6>3.0.CO;2-Z)

16 Wilkins GE, Granleese S, Hegele RG, Holden J, Anderson DW $\&$ Bondy GP. Oncogenic osteomalacia: evidence for a humoral phosphaturic factor. Journal of Clinical Endocrinology and Metabolism 199580 1628-1634. (https://doi.org/10.1210/jcem.80.5.7745010)

17 David K, Revesz T, Kratimenos G, Krausz T \& Crockard HA. Oncogenic osteomalacia associated with a meningeal phosphaturic mesenchymal tumour. Journal of Neurologicalsurgery $1996 \mathbf{8 4}$ 288-292.

18 Kim YG, Choi YS, Lee SC \& Ryu DM. Tumour-induced osteomalacia associated with lesions in the oral and maxillofacial region: report of two cases. Journal of Oral and Maxillofacial Surgery 199654 1352-1357. (https://doi.org/10.1016/s0278-2391(96)90497-8)

19 Avila NA, Skarulis M, Rubino DM \& Doppman JL. Oncogenic osteomalacia: lesion detection by MR skeletal survey. American Journal of Roentgenology 1996167 343-345. (https://doi.org/10.2214/ ajr.167.2.8686600)

20 Yang IM, Park YK, Hyun YJ, Kim DY, Woo JT, Kim SW, Kim JW, Kim YS \& Choi YK. Oncogenic osteomalacia caused by a phosphaturic mesenchymal tumour of the oral cavity: a case report. Korean Journal of Internal Medicine 199712 89-95. (https://doi. org/10.3904/kjim.1997.12.1.89)

21 Gonzalez-Compta X, Manos-Pujol M, Foglia-Fernandez M, Peral E, Condom E, Claveguera T \& Dicenta-Sousa M. Oncogenic osteomalacia: case report and review of head and neck associated tumours. Journal of Laryngology and Otology 1998112 389-392. (https://doi.org/10.1017/s0022215100140551)

22 Ohashi K, Ohnishi T, Ishikawa T, Tani H, Uesugi K \& Takagi M. Oncogenic osteomalacia presenting as bilateral stress fractures of the tibia. Skeletal Radiology 199928 46-48. (https://doi.org/10.1007/ s002560050471)

23 Clunie GPR, Fox PE \& Stamp TCB. Four cases of acquired hypophosphatemic (oncogenic) osteomalacia. Problems of diagnosis, treatment and long-term management. Rheumatology 200039 1415-1421. (https://doi.org/10.1093/ rheumatology/39.12.1415)

24 Sandhu FA \& Martuza RL. Craniofacial hemangiopericytoma associated with oncogenic osteomalacia: case report. Journal of Neuro-Oncology 200046 241-247. (https://doi. org/10.1023/a:1006352106762)

25 Reyes-Mugica M, Arnsmeier SL, Backeljauw PF, Persing J, Ellis B \& Carpenter TO. Phosphaturic mesenchymal tumour-induced rickets. Pediatric and Developmental Pathology 20003 61-69. (https://doi. org/10.1007/s100240050008)

26 Kawai Y, Morimoto S, Sakaguchi K, Yoshino H, Yotsui T, Hirota S, Inohara H, Nakagawa T, Hattori K, Kubo T, et al. Oncogenic osteomalacia secondary to nasal tumour with decreased urinary excretion of cAMP. Journal of Bone and Mineral Metabolism 200119 61-64. (https://doi.org/10.1007/s007740170062)

27 John MR, Wickert H, Zaar K, Jonsson KB, Grauer A, Ruppersberger P, Schmidt-Gayk H, Murer H, Ziegler R \& Blind E. A case of neuroendocrine oncogenic osteomalacia associated with a PHEX and fibroblast growth factor-23 expressing sinusoidal malignant schwannoma. Bone 200129 393-402. (https://doi.org/10.1016/ s8756-3282(01)00586-5)

28 Reis-Filho JS, Paiva ME \& Lopes JM. August 2003: 47-year-old female with a 7-year history of osteomalacia and hypophosphatemia. Brain Pathology 200414 111-112, 115. (https://doi. org/10.1111/j.1750-3639.2004.tb00505.x)

29 Fuentealba C, Pinto D, Ballesteros F, Pacheco D, Boettiger O, Soto N, Fernandez W, Gabler F, Gonzales G \& Reginato AJ. Oncogenic hypophosphatemic osteomalacia associated with a nasal hemangiopericytoma. Journal of Clinical Rheumatology 20039 373-379. (https://doi.org/10.1097/01.rhu.0000101906.15276.ed)

30 Ungari C, Rocchi G, Rinna C, Agrillo A, Lattanzi A \& Pagnoni M. Hypophosphaturic mesenchymal tumour of the ethmoid associated with oncogenic osteomalacia. Journal of Craniofacial Surgery 200415 523-527.

31 Dupond JL, Mahammedi H, Magy N, Blagosklonov O, MeauxRuault N \& Kantelip B. Detection of a mesenchymal tumor responsible for hypophosphatemic osteomalacia using FDG-PET. European Journal of Internal Medicine 200516 445-446. (https://doi. org/10.1016/j.ejim.2005.07.003)

32 Kaylie DM, Jackson CG \& Gardner EK. Oncogenic osteomalacia caused by phosphaturic mesenchymal tumor of the temporal bone. Otolaryngology-Head and Neck Surgery 2006135 653-654. (https://doi. org/10.1016/j.otohns.2005.03.086)

33 Inokuchi G, Tanimoto H, Ishida H, Sugimoto T, Yamauchi M, Miyauchi A \& Nibu K. A paranasal tumor associated with tumorinduced osteomalacia. Laryngoscope 2006116 1930-1933. (https:// doi.org/10.1097/01.mlg.0000231295.67060.89)

34 Yoshioka K, Nagata R, Ueda M, Yamaguchi T, Konishi Y, Hosoi M, Inoue T, Yamanaka K, Iwai Y \& Sato T. Phosphaturic mesenchymal tumor with symptoms related to osteomalacia that appeared one year after tumorectomy. Internal Medicine 200645 1157-1160. (https://doi.org/10.2169/internalmedicine.45.1797)

35 Koriyama N, Nishimoto K, Kodama T, Nakazaki M, Kurono Y, Yoshida H \& Tei C. Oncogenic osteomalacia in a case with a maxillary sinus mesenchymal tumor. American Journal of the Medical Sciences 2006332 142-147. (https://doi.org/10.1097/00000441200609000-00010)

36 Elston MS, Stewart IJ, Clifton-Bligh R \& Conaglen JV. A case of oncogenic osteomalacia with preoperative secondary hyperparathyroidism: description of the biochemical response of FGF23 to octreotide therapy and surgery. Bone 200740 236-241. (https://doi.org/10.1016/j.bone.2006.07.027)

37 Beech TJ, Rokade A, Gittoes N \& Johnson AP. A hemangiopericytoma of the ethmoid sinus causing oncogenic osteomalacia: a case report and review of the literature. International Journal of Oral and

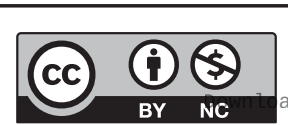

This work is licensed under a Creative Commons Attribution-NonCommercial 4.0 International License. ded from Bioscientifica com at 04/26/2023 02:16:47PM 
Maxillofacial Surgery 200736 956-958. (https://doi.org/10.1016/j ijom.2007.03.005)

38 Ahn JM, Kim HJ, Cha CM, Kim J, Yim SG \& Kim HJ. Oncogenic osteomalacia: induced by tumour, cured by surgery. Oral Surgery, Oral Medicine, Oral Pathology, Oral Radiology, and Endodontics 2007103 636-641. (https://doi.org/10.1016/j.tripleo.2005.12.027)

39 Uramoto N, Furukawa M \& Yoshizaki T. Malignant phosphaturic mesenchymal tumor, mixed connective tissue variant of the tongue. Auris, Nasus, Larynx 200936 104-105. (https://doi.org/10.1016/j. anl.2008.01.003)

40 Lewiecki EM, Urig EJ \& Williams RC. Tumor-induced osteomalacia: lessons learned. Arthritis and Rheumatism $2008 \mathbf{5 8}$ 773-777. (https:// doi.org/10.1002/art.23278)

41 Kenealy H, Holdaway I \& Grey A. Occult nasal sinus tumours causing oncogenic osteomalacia. European Journal of Internal Medicine 200819 516-519. (https://doi.org/10.1016/j.ejim.2008.01.011)

42 Yun KI, Kim DH \& Pyo SW. A phosphaturic mesenchymal tumor of the floor of the mouth with oncogenic osteomalacia: report of a case. Journal of Oral and Maxillofacial Surgery 200967 402-405. (https:// doi.org/10.1016/j.joms.2008.01.007)

43 Woo VL, Landesberg R, Imel EA, Singer SR, Folpe AL, Econs MJ, Kim T, Harik LR \& Jacobs TP. Phosphaturic mesenchymal tumor, mixed connective tissue variant, of the mandible: report of a case and review of the literature. Oral Surgery, Oral Medicine, Oral Pathology, Oral Radiology, and Endodontics 2009108 925-932. (https:// doi.org/10.1016/j.tripleo.2009.07.005)

44 Savage CR \& Zimmer LA. Oncogenic osteomalacia from pterygopalatine fossa mass. Journal of Laryngology and Otology 2009 123 1052-1054. (https://doi.org/10.1017/S0022215109004927)

45 Kurien R, Manipadam MT \& Rupa V. Oncogenic osteomalacia in a patient with an ethmoid sinus tumour. Journal of Laryngology and Otology 2010124 799-803. (https://doi.org/10.1017/ S0022215109992313)

46 Gupta R, Sharma A, Ksh A, Khadgawat R \& Dinda AK. Phosphaturic mesenchymal tumour of the sinonasal tract. Acta Endocrinologica (Bucharest) 20095 537-541. (https://doi.org/10.4183/aeb.2009.537)

47 Gore MO, Welch BJ, Geng W, Kabbani W, Maalouf NM, Zerwekn JE, Moe OW \& Sakhaee K. Renal phosphate wasting due to tumorinduced osteomalacia: a frequently delayed diagnosis. Kidney International 200976 342-347. (https://doi.org/10.1038/ ki.2008.355)

48 Kobayashi K, Nakao K, Kawai K, Ito K, Hukumoto S, Asakage T, Oota S \& Motoi R. Tumor-induced osteomalacia originating from the temporal bone: a case report. Head and Neck 201133 1072-1075. (https://doi.org/10.1002/hed.21355)

49 Shelekhova KV, Kazakov DV \& Michal M. Sinonasal phosphaturic mesenchymal tumor (mixed connective tissue variant): report of 2 cases. American Journal of Surgical Pathology 201034 596-597. (https://doi.org/10.1097/PAS.0b013e3181d594fa)

50 Pedrazzoli M, Colletti G, Ferrari M, Rossetti G, Moneghini L \& Autelitano L. Mesenchymal phosphaturic neoplasm in the maxillary sinus: a case report. International Journal of Oral and Maxillofacial Surgery 201039 1027-1032. (https://doi.org/10.1016/j. ijom.2010.04.039)

51 Mori Y, Ogasawara T, Motoi T, Shimizu Y, Chikazu D, Tamura K, Fukumoto S \& Takato T. Tumor-induced osteomalacia associated with a maxillofacial tumor producing fibroblast growth factor 23: report of a case and review of the literature. Oral Surgery, Oral Medicine, Oral Pathology, Oral Radiology, and Endodontics 2010109 e57-e63. (https:// doi.org/10.1016/j.tripleo.2009.10.052)

52 Parshwanath HA, Kulkarni PR, Rao R, Joshi SK \& Patil P. Phosphaturic mesenchymal tumor of ethmoid sinus. Indian Journal of Pathology and Microbiology 201053 384-385. (https://doi.org/10.4103/03774929.64317)

53 Battoo AJ, Salih S, Unnikrish AG, Jojo A, Bahadur S, Iyer S \& Kuriakose MA. Oncogenic osteomalacia from nasal cavity giant cell tumor. Head and Neck 201234 454-457. (https://doi. org/10.1002/hed.21562)

54 Arnaoutakis D \& Naseri I. Sinonasal phosphaturic mesenchymal tumor: a rare and misinterpreted entity. Journal of Neurological Surgery Reports 201576 e233-e238. (https://doi. org/10.1055/s-0035-1562852)

55 Peters KB, McLendon R, Morse MA \& Vredenburgh JJ. Treatment of recurrent intracranial hemangiopericytoma with SRC-related tyrosine kinase targeted therapy: a case report. Case Reports in Oncology 20103 93-97. (https://doi.org/10.1159/000307468)

56 Akhter M, Sugrue PA, Bains R \& Khavkin YA. Oncogenic osteomalacia of the cervical spine: a rare case of curative resection and reconstruction. Journal of Neurosurgery. Spine 201114 453-456. (https://doi.org/10.3171/2010.11.SPINE09750)

57 Xian-Ling W, Jian-Ming B, Wen-wen Z, Zhao-Hui L, Jing-Tao D, Ju-Ming L \& Yi-Ming M. Osteomalacia caused by tumors in facies cranii mimicking rheumatoid arthritis. Rheumatology International 201232 2573-2576. (https://doi.org/10.1007/s00296-011-2018-4)

58 Guglielmi G, Bisceglia M, Scillitani A \& Folpe AL. Oncogenic osteomalacia due to phosphaturic mesenchymal tumor of the craniofacial sinuses. Clinical Cases in Mineral and Bone Metabolism $2011845-49$.

59 Uno T, Kawai K, Kunii N, Fukumoto S, Shibahara J, Motoi T \& Saito N. Osteomalacia caused by skull base tumors: report of 2 cases. Neurosurgery 201169 E239-E244; discussion E244. (https://doi. org/10.1227/NEU.0b013e31821867f7)

60 Andreupoulou P, Dumitrescu CE, Kelly MH, Brillante BA, Peck CMC, Wodajo FM, Chang R \& Collins MT. Selective venous catheterization for the localization of phosphaturic mesenchymal tumors. Journal of Bone and Mineral Research 20116 1295-1302.

61 Bergwitz C, Collins MT, Kamath RS \& Rosenberg AE. Case 33-2011: a 56-year-old man with hypophosphatemia. New England Journal of Medicine 2011365 1625-1635. (https://doi.org/10.1056/ NEJMcpc1104567)

62 Monappa V, Naik AM, Mathew M, Rao L, Rao SK, Ramachandra L \& Padmapriya J. Phosphaturic mesenchymal tumour of the mandible - the useful criteria for a diagnosis on fine needle aspiration cytology. Cytopathology 201425 54-56. (https://doi.org/10.1111/ cyt.12030)

63 Chokyu I, Ishibashi K, Goto T \& Ohata K. Oncogenic osteomalacia associated with mesenchymal tumor in the middle cranial fossa: a case report. Journal of Medical Case Reports 20126 181. (https://doi. org/10.1186/1752-1947-6-181)

64 Chiam P, Tan HC, Bee YM \& Chandran M. Oncogenic osteomalacia hypophosphatemic spectrum from benignancy to malignancy. Bone 201353 182-187. (https://doi.org/10.1016/j.bone.2012.11.040)

65 Cho S, Do NY, Yu SW \& Choi JY. Nasal hemangiopericytoma causing oncogenic osteomalacia. Clinical and Experimental Otolaryngology 20123 173-176.

66 Burnand H, Samuels A, Hagan I, Sawant N \& Mutimer J. Bilateral subtrochanteric fractures in tumor-induced osteomalacia caused by a nasal hemangiopericytoma. Hip International 201222 227-229. (https://doi.org/10.5301/HIP.2012.9235)

67 Brandwein-Gensler M \& Siegal GP. Striking pathology gold: a singular experience with daily reverberations: sinonasal hemangiopericytoma (glomangiopericytoma) and oncogenic osteomalacia. Head and Neck Pathology 20126 64-74. (https://doi.org/10.1007/s12105-012-0337-8)

68 Munoz J, Ortega RM, Celzo F \& Donthireddy V. Tumour-induced osteomalacia. BMJ Case Reports 20122012 bcr0320125975. (https:// doi.org/10.1136/bcr.03.2012.5975)

69 Chang CV, Conde SJ, Luvizotto RAM, Nunes VS, Bonates MC, Felicio AC, Lindsey SC, Moraes FH, Tagliarini JV, Mazeto GMFS, et al. Oncogenic osteomalacia: loss of hypophosphatemia might be the key to avoid misdiagnosis. Arquivos Brasileiros de Endocrinologia e Metabologia 201256 570-573. (https://doi.org/10.1590/S000427302012000800018)

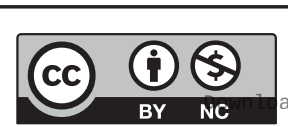

This work is licensed under a Creative Commons Attribution-NonCommercial 4.0 International License. Aed from Bioscientifica com at $04 / 26 / 2023$ 02:16:47PM 
70 Jiang Y, Xia WB, Xing XP, Silva BC, Li M, Wang O, Zhang HB, Li F, Jing HL, Zhong DR, et al. Tumor-induced osteomalacia: an important cause of adult-onset hypophosphatemic osteomalacia in China: report of 39 cases and review of the literature. Journal of Bone and Mineral Research 201227 1967-1975. (https://doi.org/10.1002/ jbmr.1642)

71 Fatani HA, Sunbuli M, Lai SY \& Bell D. Phosphaturic mesenchymal tumor: a report of 6 patients treated at a single institution and comparison with reported series. Annals of Diagnostic Pathology 2013 17 319-321. (https://doi.org/10.1016/j.anndiagpath.2012.06.005)

72 Mathis DA, Stehel EJ, Beshay JE, Mickey BE, Folpe AL \& Raisanen J. Intracranial phosphaturic mesenchymal tumors: report of 2 cases. Journal of Neurosurgery 2013118 903-907. (https://doi. org/10.3171/2012.12.JNS12598)

73 Tarasova VD, Trepp-Carrasco AG, Thompson R, Recker RR, Chong WH, Collins MT \& Armas LA. Successful treatment of tumor-induced osteomalacia due to an intracranial tumor by fractionated stereotactic radiotherapy. Journal of Clinical Endocrinology and Metabolism 201398 4267-4272. (https://doi.org/10.1210/jc.2013-2528)

74 Papierska L, Ćwikła JB, Misiorowski W, Rabijewski M, Sikora K \& Wanyura H. Unusual case of phosphaturic mesenchymal tumor. Polskie Archiwum Medycyny Wewnetrznej 2013123 255-256. (https:// doi.org/10.20452/pamw.1738)

75 Lee GG, Dhong HJ, Park YS \& Ko YH. Sinonasal glomangiopericytoma causing oncogenic osteomalacia. Clinical and Experimental Otorhinolaryngology 20147 145-148. (https://doi. org/10.3342/ceo.2014.7.2.145)

76 Allevi F, Rabbiosi D, Mandala M \& Colletti G. Mesenchymal phosphaturic tumour: early detection of recurrence. BMJ Case Reports 20142014 bcr2013202827. (https://doi.org/10.1136/bcr-2013202827)

77 Annamalai AK, Sampathkumar K, Kane S, Shetty NS, Kulkarni S, Rangarajan V, Purandare N, Pai PS, Mahuvakar AD, Shanthi R, et al. Needles in the haystack - synchronous multifocal tumor-induced osteomalacia. Journal of Clinical Endocrinology and Metabolism 2016 101 390-393. (https://doi.org/10.1210/jc.2015-3854)

78 Okamiya T, Takahashi K, Kamada H, Hirato J, Motoi T, Fukumoto S \& Chikamatsu K. Oncogenic osteomalacia caused by an occult paranasal sinus tumor. Auris, Nasus, Larynx 201542 167-169. (https://doi.org/10.1016/j.anl.2014.10.001)

79 Mok Y, Lee JC, Lum JHY \& Petersson F. From epistaxis to bone pain - report of two cases illustrating the clinicopathological spectrum of phosphaturic mesenchymal tumor with fibroblast growth factor receptor 1 immunohistochemical and cytogenetic analyses. Histopathology 201668 925-930. (https://doi.org/10.1111/ his.12872)

80 Fernandez-Cooke E, Cruz-Rojo J, Gallego C, Romance AI, MosquedaPena R, Almaden Y \& del Pozo JS. Tumor-induced rickets in a child with a central giant cell granuloma: a case report. Pediatrics 2015135 e1518-e1523. (https://doi.org/10.1542/peds.2014-2218)

81 Fathalla H, Cusimano M, Di leva A, Karamchandani J, Fung R \& Kovacs K. Osteomalacia-inducing tumors of the brain: a case report, review and a hypothesis. World Neurosurgery 201584 189.e1-189.e5. (https://doi.org/10.1016/j.wneu.2015.02.030)

82 Ray S, Chakraborty PP, Biswas K, Ghosh S, Mukhopadhyay S \& Chowdhury S. A case of oncogenic osteomalacia due to occult nasal sinus tumor. Clinical Cases in Mineral and Bone Metabolism 201512 65-68. (https://doi.org/10.11138/ccmbm/2015.12.1.065)

83 Qari H, Hamao-Sakamoto A, Fuselier C, Cheng YSL, Kessler H \& Wright J. Phosphaturic mesenchymal tumor: 2 new oral cases and review of 53 cases in the head and neck. Head and Neck Pathology 201610 192-200. (https://doi.org/10.1007/s12105015-0668-3)

84 Wasserman JK, Purgina B, Lai CK, Gravel D, Mahaffey A, Bell D \& Chiosea SI. Phosphaturic mesenchymal tumor involving the head and neck: a report of five cases with FGFR1 fluorescence in situ hybridization analysis. Head and Neck Pathology 201610 279-285. (https://doi.org/10.1007/s12105-015-0678-1)

85 Mani MK \& Panigrahi MK. Unusual calvarial tumour-oncogenic osteomalacia. British Journal of Neurosurgery 201731 495-496. (https://doi.org/10.3109/02688697.2016.1161165)

$86 \mathrm{Yu}$ WJ, He JW, Fu WZ, Wang C \& Zhang ZL. Reports of 17 Chinese patients with tumor-induced osteomalacia. Journal of Bone and Mineral Metabolism 201735 298-307. (https://doi.org/10.1007/ s00774-016-0756-9)

87 Takashi Y, Kinoshita Y, Ito N, Taguchi M, Takahashi M, Egami N, Tajima S, Nangaku M \& Fukumoto S. Tumor-induced osteomalacia caused by a parotid tumor. Internal Medicine 201756 535-539. (https://doi.org/10.2169/internalmedicine.56.7565)

88 Gresham MS, Shen S, Zhang YJ \& Gallagher K. Anterior skull base glomangioma-induced osteomalacia. Journal of Neurological Surgery Reports $2017 \mathbf{7 8}$ e9-e11. (https://doi. org/10.1055/s-0036-1597599)

89 Agaimy A, Michal M, Chiosea S, Petersson F, Hadravsky L, Kristiansen G, Horch RE, Schmolders J, Hartmann A, Haller F, et al. Phosphaturic mesenchymal tumors: clinicopathologic, immunohistochemical and molecular analysis of 22 cases expanding their morphologic and immunophenotypic spectrum. American Journal of Surgical Pathology 201741 1371-1380. (https://doi. org/10.1097/PAS.0000000000000890)

90 Lee JY, Park HS, Han S, Lim JK, Hong N, Park SI \& Rhee Y. Localization of oncogenic osteomalacia by systemic venous sampling of fibroblast growth factor 23. Yonsei Medical Journal $2017 \mathbf{5 8}$ 981-987. (https://doi.org/10.3349/ymj.2017.58.5.981)

91 Schober HC, Kneitz C, Fieber F, Hesse K \& Schroeder H. Selective blood sampling for FGF-23 in tumor-induced osteomalacia. Endocrinology, Diabetes and Metabolism Case Reports 201720171. (https://doi.org/10.1530/EDM-17-0006)

92 Zuo QY, Wang H, Li W, Niu XH, Huang YH, Chen J, You YH, Liu BY, Cui AM \& Deng W. Treatment and outcomes of tumorinduced osteomalacia associated with phosphaturic mesenchymal tumors: retrospective review of 12 patients. BMC Musculoskeletal Disorders 201718 403. (https://doi.org/10.1186/s12891-0171756-1)

93 Hana T, Tanaka S, Nakatomi H, Shojima M, Fukumoto S, Ikemura M \& Saito N. Definitive surgical treatment of osteomalacia induced by skull base tumor and determination of the half-life of serum fibroblast growth factor 23. Endocrine Journal 201764 1033-1039. (https://doi.org/10.1507/endocrj.EJ17-0177)

94 Chanukya GV, Mengade M, Goud J, Rao IS \& Jain A. Tumorinduced osteomalacia: a Sherlock Holmes approach to diagnosis and management. Annals of Maxillofacial Surgery 20177 143-147. (https://doi.org/10.4103/ams.ams_123_16)

95 Gonzalez G, Baudrand R, Sepulveda MF, Vucetich N, Guarda FJ, Villanueva P, Contreras O, Villa A, Salech F, Toro L, et al. Tumorinduced osteomalacia: experience from a South American academic centre. Osteoporosis International 201728 2187-2193. (https://doi. org/10.1007/s00198-017-4007-2)

96 Singh D, Chopra A, Ravina M, Kongara S, Bhatia E, Kumar N, Gupta S, Yadav S, Dabadghao P, Yadav R, et al. Oncogenic osteomalacia: role of Ga-68 DOTANOC PET/CT scan in identifying the culprit lesion and its management. British Journal of Radiology 201790 20160811. (https://doi.org/10.1259/bjr.20160811)

97 Pelletier K, Troyanov S, Guite JF, Sainte-Marie LG, Roberge D \& Lessard M. Localization of ectopic fibroblast growth factor 23 production in tumor-induced osteomalacia using selective venous samplings. Clinical Nephrology 201787 107-110. (https://doi. org/10.5414/CN108981)

98 Mumford E, Marks J, Wagner T, Gallimore A, Gane S \& Walsh SB. Oncogenic osteomalacia: diagnosis, localisation, and cure. Lancet Oncology 201819 e365. (https://doi.org/10.1016/S14702045(18)30276-6) 
99 Villepelet A, Casiraghi O, Temam S \& Moya-Plana A. Ethmoid tumor and oncogenic osteomalacia: case report and review of the literature. European Annals of Otorhinolaryngology, Head and Neck Diseases 2018135 365-369. (https://doi.org/10.1016/j. anorl.2018.07.001)

100 Pelo S, Gasparini G, Garagiola U, D’Amato G, Saponaro G, Doneddu P, Todaro M \& Moro A. Phosphaturic mesenchymal tumor, an unusual localization in head and neck. Journal of Surgical Case Reports 20182018 rjy091. (https://doi.org/10.1093/jscr/rjy091)

101 He Q, Xu Z, Zhang B, Hu W \& Zhang X. Tumor-induced osteomalacia caused by a parotid basal cell adenoma detected by 68Ga-DOTANOC PET/CT. Clinical Nuclear Medicine $2018 \mathbf{4 3}$ e198-e199. (https://doi.org/10.1097/RLU.0000000000002076)

102 Wu H, Bui MM, Zhou L, Li D, Zhang H \& Zhong D. Phosphaturic mesenchymal tumor with an admixture of epithelial and mesenchymal elements in the jaws: clinicopathological and immunohistochemical analysis of 22 cases with literature review. Modern Pathology 201932 189-204. (https://doi.org/10.1038/s41379018-0100-0)

103 Ding J, Hu G, Wang L, Li F \& Huo L. Increased activity due to fractures does not significantly affect the accuracy of 68Ga-DOTATATE PET/CT in the detection of culprit tumor in the evaluation of tumor-induced osteomalacia. Clinical Nuclear Medicine 201843 880-886. (https://doi.org/10.1097/ RLU.0000000000002290)

104 Mishra T, Desouza MA, Patel K \& Mazumdar GA. Phosphaturic mesenchymal tumors involving skull bones: report of two rare cases. Asian Journal of Neurosurgery 201914 253-255. (https://doi. org/10.4103/ajns.AJNS_176_17)

105 Li J, Huang Y, Yang F, Zhang Q, Chen D \& Wang Q. Sinonasal hemangiopericytoma caused hypophosphatemic osteomalacia: a case report. Medicine 201897 e13849. (https://doi.org/10.1097/ MD.0000000000013849)

106 Acharya RP, Won AM, Moon BS, Flint JH, Roubaud MS, Williams MD, Hessel AC, Murphy Jr WA, Chambers MS \& Gagel RF. Tumor-induced hypophosphatemic osteomalacia caused by a mesenchymal tumor of the mandible managed by a segmental mandibulectomy and microvascular reconstruction with a free fibula flap. Head and Neck 201941 E93-E98. (https://doi.org/10.1002/hed.25657)

107 Kurien R, Rupa V \& Thomas M. Varied presentation of sinonasal phosphaturic mesenchymal tumour: report of a case series with follow-up. European Archives of Oto-Rhino-Laryngology 2019276 1677-1684. (https://doi.org/10.1007/s00405-019-05341-8)

108 Paul J, Cherian KE, Kapoor N \& Paul TV. Treating osteoporosis: a near miss in an unusual case of FGF-23 mediated bone loss. BMJ Case Reports 201912 e228375. (https://doi.org/10.1136/bcr-2018228375)

109 Pal R, Bhadada SK, Singhare A, Bhansali A, Kamalanathan S, Chadha M, Chauhan P, Sood A, Dhiman V, Sharma DC, et al. Tumor-induced osteomalacia: experience from three tertiary care centers in India. Endocrine Connections 20198 266-276. (https://doi. org/10.1530/EC-18-0552)

110 Jadhav S, Kasaliwal R, Lele V, Rangarajan V, Chandra P, Shah H, Malhotra G, Jagtap VS, Budyal S, Lila AR, et al. Functional imaging in primary tumour-induced osteomalacia: relative performance of FDG PET/CT vs somatostatin receptor-based functional scans: a series of nine patients. Clinical Endocrinology 201481 31-37. (https://doi. org/10.1111/cen.12426)

111 Agrawal K, Bhadada S, Mittal BR, Shukla J, Sood A, Bhattacharya A \& Bhansali A. Comparison of 18F-FDG and 68Ga DOTATATE PET/ CT in localization of tumor causing oncogenic osteomalacia. Clinical Nuclear Medicine 201540 e6-e10. (https://doi.org/10.1097/ RLU.0000000000000460)

112 El-Maouche D, Sadowski SM, Papadakis GZ, Guthrie L, CottleDelisle C, Merkel R, Millo C, Chen CC, Kebebew E \& Collins MT. 68Ga-DOTATATE for tumor localization in tumor-induced osteomalacia. Journal of Clinical Endocrinology and Metabolism 2016 101 3575-3581. (https://doi.org/10.1210/jc.2016-2052)

113 Basu S \& Fargose P. 177Lu-DOTATATE PRRT in recurrent skullbase phosphaturic mesenchymal tumor causing osteomalacia: a potential application of PRRT beyond neuroendocrine tumors. Journal of Nuclear Medicine Technology 201644 248-250. (https://doi. org/10.2967/jnmt.116.177873)

114 Kane SV, Kakkar A, Oza N, Sridhar E \& Pai PS. Phosphaturic mesenchymal tumor of the nasal cavity and paranasal sinuses: a clinical curiosity presenting a diagnostic challenge. Auris, Nasus, Larynx 201845 377-383. (https://doi.org/10.1016/j. anl.2017.05.006)

115 Feng J, Jiang Y, Wang O, Li M, Xing X, Huo L, Li F, Yu W, Zhong DR Jin J, et al. The diagnostic dilemma of tumor induced osteomalacia: a retrospective analysis of 144 cases. Endocrine Journal 201764 675-683. (https://doi.org/10.1507/endocrj.EJ16-0587)

116 Khosravi A, Cutler CM, Kelly MH, Chang R, Royal RE, Sherry RM, Wodajo FM, Fedarko NS \& Collins MT. Determination of the elimination half-life of fibroblast growth factor-23. Journal of Clinical Endocrinology and Metabolism 200792 2374-2377. (https://doi. org/10.1210/jc.2006-2865)

117 Takeuchi Y, Suzuki H, Ogura S, Imai R, Yamazaki Y, Yamashita T, Miyamoto Y, Okazaki H, Nakamura K, Nakahara K, et al. Venous sampling for fibroblast growth factor-23 confirms preoperative diagnosis of tumor-induced osteomalacia. Journal of Clinical Endocrinology and Metabolism 200489 3979-3982. (https://doi. org/10.1210/jc.2004-0406)

118 Minisola S, Peacock M, Fukumoto S, Cipriani C, Pepe J, Tella SH \& Collins MT. Tumour-induced osteomalacia. Nature Reviews Disease Primers 20173 17044. (https://doi.org/10.1038/nrdp.2017.44)

119 Reubi JC, Waser B, Schaer JC \& Laissue JA. Somatostatin receptor sst1-sst5 expression in normal and neoplastic human tissues using receptor autoradiography with subtype-selective ligands. European Journal of Nuclear Medicine 200128 836-846. (https://doi. org/10.1007/s002590100541)

120 de Beur SMJ, Streeten EA, Civelek AC, McCarthy EF, Uribe L, Marx SJ, Onobrakpeya O, Raisz LG, Watts NB, Sharon M, et al. Localisation of mesenchymal tumours by somatostatin receptor imaging. Lancet 2002359 761-763. (https://doi.org/10.1016/S01406736(02)07846-7)

121 Kimizuka T, Ozaki Y \& Sumi Y. Usefulness of $201 \mathrm{Tl}$ and 99m Tc MIBI scintigraphy in a case of oncogenic osteomalacia. Annals of Nuclear Medicine 200418 63-67. (https://doi.org/10.1007/BF02985616)

122 Dupond JL, Mahammedi H, Prie D, Collin F, Gil H, Blagosklonov O, Ricbourg B, Meaux-Ruault N \& Kantelip B. Oncogenic osteomalacia: diagnostic importance of fibroblast growth factor 23 and F-18 fluorodeoxyglucose PET/CT scan for the diagnosis and follow-up in one case. Bone 200536 375-378. (https://doi.org/10.1016/j. bone.2005.01.001)

123 Breer S, Brunkhorst T, Beil FT, Peldschus K, Heiland M, Klutmann S, Barvencik F, Zustin J, Gratz KF \& Amling M. 68Ga DOTATATE PET/ CT allows tumor localization in patients with tumor-induced osteomalacia but negative 111In-octreotide SPECT/CT. Bone 201464 222-227. (https://doi.org/10.1016/j.bone.2014.04.016)

124 Zhang J, Zhu Z, Zhong D, Dang Y, Xing H, Du Y, Jing H, Qiao Z, Xing $\mathrm{X}$, Zhuang $\mathrm{H}$, et al. 68Ga DOTATATE PET/CT is an accurate imaging modality in the detection of culprit tumors causing osteomalacia. Clinical Nuclear Medicine 201540 642-646. (https:// doi.org/10.1097/RLU.0000000000000854)

125 Seufert J, Ebert K, Müller J, Eulert J, Hendrich C, Werner E, Schütze N, Schulz G, Kenn W, Richtmann H, et al. Octreotide therapy for tumorinduced osteomalacia. New England Journal of Medicine 2001345 1883-1888. (https://doi.org/10.1056/NEJMoa010839)

126 Ovejero D, El-Maouche D, Brillante BA, Khosravi A, Gafni RI \& Collins MT. Octreotide is ineffective in treating tumor-induced osteomalacia: results of a short-term therapy. Journal of Bone and

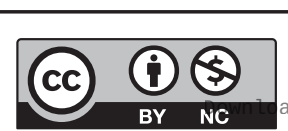

This work is licensed under a Creative Commons Attribution-NonCommercial 4.0 International License. ded from Bioscientifica com at 04/26/2023 02:16:47PM 
Mineral Research 201732 1667-1671. (https://doi.org/10.1002/ jbmr.3162)

127 Geller JL, Khosravi A, Kelly MH, Riminucci M, Adams JS \& Collins MT. Cinacalcet in the management of tumor-induced osteomalacia. Journal of Bone and Mineral Research 200722 931-937. (https://doi.org/10.1359/jbmr.070304)
128 Cives M \& Strosberg J. Radionuclide therapy for neuroendocrine tumors. Current Oncology Reports 201719 9. (https://doi.org/10.1007/ s11912-017-0567-8)

129 Weidner N. Review and update: oncogenic osteomalaciarickets. Ultrastructural Pathology 199115 317-333. (https://doi org/10.3109/01913129109016242)

Received in final form 15 August 2019

Accepted 9 September 2019

Accepted Preprint published online 9 September 2019 Attribution-NonCommercial 4.0 International License. 